This item was submitted to Loughborough's Research Repository by the author.

Items in Figshare are protected by copyright, with all rights reserved, unless otherwise indicated.

\title{
Reinforcement learning-based fixed-time trajectory tracking control for uncertain robotic manipulators with input saturation
}

\section{PLEASE CITE THE PUBLISHED VERSION}

https://doi.org/10.1109/TNNLS.2021.3116713

\section{PUBLISHER}

Institute of Electrical and Electronics Engineers

\section{VERSION}

AM (Accepted Manuscript)

\section{PUBLISHER STATEMENT}

(c) 2021 IEEE. Personal use of this material is permitted. Permission from IEEE must be obtained for all other uses, in any current or future media, including reprinting/republishing this material for advertising or promotional purposes, creating new collective works, for resale or redistribution to servers or lists, or reuse of any copyrighted component of this work in other works.

\section{LICENCE}

\section{All Rights Reserved}

\section{REPOSITORY RECORD}

Cao, Shengjie, Liang Sun, Jingjing Jiang, and Zongyu Zuo. 2021. "Reinforcement Learning-based Fixed-time Trajectory Tracking Control for Uncertain Robotic Manipulators with Input Saturation”. Loughborough University. https://hdl.handle.net/2134/16691437.v1. 


\title{
Reinforcement Learning Based Fixed-Time Trajectory Tracking Control for Uncertain Robotic Manipulators with Input Saturation
}

\author{
Shengjie Cao, Liang Sun, Member, IEEE, Jingjing Jiang, Member, IEEE, Zongyu Zuo, Senior Member, IEEE
}

\begin{abstract}
A fixed-time trajectory tracking control method for uncertain robotic manipulators with input saturation based on reinforcement learning $(R L)$ is studied. The designed reinforcement learning control algorithm is implemented by radial basis function (RBF) neural network, in which the actor neural network is used to generate the control strategy and the critic neural network is used to evaluate the execution cost. A new non-singular fast terminal sliding mode technique is used to ensure the convergence of tracking error in fixed time, and the upper bound of convergence time is estimated. To solve the saturation problem of an actuator, a nonlinear anti-windup compensator is designed to compensate for the saturation effect of the joint torque actuator in real time. Finally, the stability of the closed-loop system based on Lyapunov candidate is analyzed, and the timing convergence of the closed-loop system is proved. Simulation and experimental results show the effectiveness and superiority of the proposed control law.
\end{abstract}

Index Terms-Uncertain robotic manipulators, trajectory tracking control, reinforcement learning, fixed-time control, input saturation, non-singular terminal sliding mode control

\section{INTRODUCTION}

$\mathbf{R}$ OBOTIC manipulators are widely used in fields of military, manufacturing industry, medical and other hazardous environment fields. The trajectory tracking technology of the robotic arm is always the hot research direction. The movement of the robotic system according to the preset joint trajectory is the key to realize complex tasks. However, due to the uncertainty of the dynamic model, coupling effect and unknown external disturbances, it is very difficult for the manipulator to track the trajectory accurately and quickly. Therefore, it is urgent to explore an effective control technology [1] [2].

In recent years, many researchers have focus on the design of finite time control algorithms for mechanical systems

This work was supported by the National Natural Science Foundation of China (Nos. 61903025, 62073019), the China Scholarship Council (No. 201906465028), and the Fundamental Research Funds for the Central Universities (No. FRF-BD-19-002A).

S. Cao and L. Sun are with the Key Laboratory of Knowledge Automation for Industrial Processes of Ministry of Education, School of Automation and Electrical Engineering, University of Science and Technology Beijing, Beijing 100083, China; Institute of Artificial Intelligence, University of Science and Technology Beijing, Beijing 100083, China (e-mail: csj19970720@163.com; liangsundustb.edu.cn)

J. Jiang is with the Department of Aeronautical and Automotive Engineering, Loughborough University, Leicestershire, England LE11 3TU, United Kingdom.(e-mail: J.Jiang2 (lboro.ac.uk)

Z. Zuo is with the Seventh Research Division, School of Automation Science and Electrical Engineering, Beihang University, Beijing 100191, China (e-mail: zzybobby@buaa. edu. cn) described by the Euler-Lagrange equation. In [3], a robust finite-time control approach for robot systems was developed based on a finite-time Lyapunov stability principle. In [4] and [5], a non-singular terminal sliding mode controller based on an extended state observer was designed to realize the convergence of trajectory tracking errors in finite time. Based on a polynomial reference trajectory, a simple linear sliding surface was proposed in [6]. A novel non-singular integral terminal sliding mode surface was formulated in [7], Moreover, a new adaptive parameter tuning law was also developed by making good use of the system tracking errors and the adaptive parameter estimation errors. An adaptive finite-time controller was proposed in [8] based on a second-order terminal sliding mode. All of the above studies limit the convergence time to a certain range through finite-time control, but all of them depend on the initial state of the system.

In order to get rid of the constraints of the initial state and further improve the convergence performance of the closed loop system within the finite time, the fixed-time stability extended from the finite-time stability [9] was developed in recent years, and many theoretical results [11] [10] and practical applications [12] [13] have been made. On the basis of previous studies [6], the finite-time convergence is further improved to the fixed time convergence in [14], with effectively reducing the convergence time of tracking errors. In [15], a new cascade control structure, based on a fixed-time distributed observer, was developed to achieve the fixed-time consensus tracking control. In [16], based on the fixed-time control technique and the uniform exact difference method, a new fixed-time tracking control with a novel secondorder sliding mode super-twisting-like structure adaptive fixedtime disturbance observer was proposed. In [17], the fixedtime control problem was addressed for rigid spacecraft with actuator saturation and faults. The fixed-time stabilisation has been discussed for a class of uncertain nonholonomic systems with matched unknown time-varying nonlinear perturbations in [18]. In [19], a new version of the adaptive fixed-time convergent observer was proposed to estimate a disturbance without excessively increasing the controller gains, where the Lipschitz constant of disturbance and initial values are unknown. The robust fixed-time stabilization control problem has been investigated for generic multivariable linear systems subject to both mismatched and matched disturbances or uncertainties in [20]. All of the above methods have been proven to be useful, but all of the controller design require prior knowledge of dynamics. 
Because artificial neural networks (NN) have good general approximation capabilities. If there is no or relatively limited system dynamics knowledge, it can be used to effectively construct a controller to achieve the desired control task. Therefore, it has been widely used in the control design of uncertain nonlinear systems [21]-[24]. NN-based RL is different from supervised learning, which is a learning method that obtains training information from the environment. In recent years, it has made great progress in the field of intelligent control by combining RL with traditional control methods for reference tracking [25]-[27]. In [25], a novel virtual state-feedback tuning and value iteration RL were applied for learning linear reference model output tracking control of observable systems with unknown dynamics. In [26], the author uses RL method to improve the tracking accuracy and robustness of the $\mathrm{H}_{2}$ control. In [27], a RL control strategy, which is based on actor-critic structure, was developed to enable vibration suppression while retaining trajectory tracking. The actor-critic strategy used in [27] is a classic RL framework. The actor NN generation strategy is used to give the input of the executor, and the critic $\mathrm{NN}$ is used to evaluate the execution cost to determine whether the situation is better or worse than expected, and to define a value for measurement. Then, the actor modifies its control strategy based on the critic valuation. Through these steps, the cost function will converge to the global optimum [28]. Based on the actor-critic strategy, some excellent research results have attracted considerable attention [29]-[32]. In [29], the flight control algorithm based on RL was designed for the attitude control of small unmanned helicopters to compensate the model uncertainty only by using online flight data of the helicopter. An adaptive control algorithm was proposed in [30] for a class of robot manipulator systems with unknown functions and dead-zone input by using a RL scheme. In [31], the RL strategy of actor-critic architecture based on neural network was used to overcome the nonlinearity and intractability in the optimization process. Aiming at the single link flexible manipulator model, the RL control strategy with actor-critic structure was adopted in [32] to improve the control accuracy and robustness against the uncertain system dynamics. In addition, there are also some examples of combining actor-critic structure with traditional control methods. For example, in [33], RL is combined with a traditional PID control, which solves the shortcomings of traditional PID controllers that cannot be self-tuning online. All of the above RL control methods have been proved to be effective, but they are limited to a finite-time framework and have not been combined with a fixed-time algorithm.

This paper designs a fixed-time control algorithm for the uncertain robotic manipulators control system that combines the non-singular terminal sliding mode control with the RL method. Considering the input saturation, a nonlinear antiwindup compensator is designed to compensate for the saturation overflow effect of joint torque actuators. It is proven that all signals in the closed-loop system are bounded and the system output follows the desired reference signal. A simulation example is given to illustrate the effectiveness of the proposed method. The main advantages and contributions of this paper are summarized as follows.
1) In [34], a saturated adaptive back-stepping controller with NN approximation was proposed. However, this method does not consider the optimal control problem and ignores the cost function. In this paper, a control method based on RL is proposed, which can obtain the optimized control strategy based on state information and reduce the design cost.

2) In the above-mentioned references [29]-[32], RL is only limited to a finite time framework, but the proposed method combines reinforcement learning with a fixed time framework to reduce the convergence time and improve the steady-state accuracy of the system.

3) A nonlinear anti-windup compensator in fixed-time designing framework is designed to counteract the saturation effect of the joint torque actuator in real time.

4) Within the actor-critic RL framework, the designed controller makes the fixed-time convergence of trajectory tracking error, the parameter projection algorithm is applied to ensure the boundedness of the weight vector of $\mathrm{NN}$, which provides a rigorous argument for the stability proof of the closed-loop system.

The remainder of this paper is organized as follows. Section II presents the mathematical model and the tracking task design objectives. In Section III, a fixed-time sliding mode controller based on reinforcement learning with input saturation is designed, and the stability of the closed-loop system is proved. In Section IV, numerical simulations on two-link rigid robotic manipulators and experimental results on the joint 3 of KINOVA are presented. Conclusions and future works are discussed in Section V.

Throughout this paper, for any $\boldsymbol{x}=\left[x_{1}, x_{2}, \ldots, x_{n}\right]^{T} \in$ $\mathbb{R}^{n}$, its fractional-order vector denoted as $\operatorname{sig}^{v}(\boldsymbol{x})=$ $\left[\left|x_{1}\right|^{v} \operatorname{sign}\left(x_{1}\right), \cdots,\left|x_{n}\right|^{v} \operatorname{sign}\left(x_{n}\right)\right]^{T}$, where $\operatorname{sign}(\cdot)$ is the standard signum function. we use $\|\cdot\|$ for the Euclidean norm of vectors and the induced norm for matrices, $\lambda_{m}(A)$ and $\lambda_{M}(A)$ denote the minimum and maximum eigenvalues of a matrix $A \in \mathbb{R}^{n \times n}$, respectively.

\section{Problem Statement}

Consider the dynamic model of uncertain robotic manipulators system as [5]

$$
M(\boldsymbol{q}) \ddot{\boldsymbol{q}}+C(\boldsymbol{q}, \dot{\boldsymbol{q}}) \dot{\boldsymbol{q}}+\boldsymbol{g}(\boldsymbol{q})=\boldsymbol{\tau}(t)+\boldsymbol{d}(t)
$$

where $\boldsymbol{q}(t) \in \mathbb{R}^{n}, \dot{\boldsymbol{q}}(t) \in \mathbb{R}^{n}$ and $\ddot{\boldsymbol{q}}(t) \in \mathbb{R}^{n}$ denote the link position, velocity, and acceleration, respectively; $M(\boldsymbol{q})=M_{0}(\boldsymbol{q})+M_{\Delta}(\boldsymbol{q}) \in \mathbb{R}^{n \times n}$ is the inertia matrix; $C(\boldsymbol{q}, \dot{\boldsymbol{q}})=C_{0}(\boldsymbol{q}, \dot{\boldsymbol{q}})+C_{\Delta}(\boldsymbol{q}, \dot{\boldsymbol{q}}) \in \mathbb{R}^{n \times n}$ is the centrifugal-Coriolis matrix; $\boldsymbol{g}(\boldsymbol{q})=\boldsymbol{g}_{0}(\boldsymbol{q})+\boldsymbol{g}_{\Delta}(\boldsymbol{q}) \in \mathbb{R}^{n}$ is the potential force; $M_{0}(\boldsymbol{q}), C_{0}(\boldsymbol{q}, \dot{\boldsymbol{q}}), \boldsymbol{g}_{0}(\boldsymbol{q})$ are known, and $M_{\Delta}(\boldsymbol{q}), C_{\Delta}(\boldsymbol{q}, \dot{\boldsymbol{q}}), \boldsymbol{g}_{\Delta}(\boldsymbol{q})$ are unknown but bounded; $\boldsymbol{d}(t) \in$ $\mathbb{R}^{n}$ is the external disturbance torque; $\boldsymbol{\tau}(t) \in \mathbb{R}^{n}$ is the control input.

From the non-singular property of the matrix $M_{0}(\boldsymbol{q})$, the matrix $M_{0}(\boldsymbol{q})$ is invertible, so the model (1) can be written as

$$
\ddot{\boldsymbol{q}}=M_{0}^{-1} \boldsymbol{\tau}+M^{-1} \boldsymbol{d}+\boldsymbol{l}(t)
$$

where $\boldsymbol{l}(t)=M^{-1}[-C(\boldsymbol{q}, \dot{\boldsymbol{q}}) \dot{\boldsymbol{q}}-\boldsymbol{g}(\boldsymbol{q})]+\bar{M}_{\Delta} \boldsymbol{\tau}$ is the modeling uncertainty, and $\bar{M}_{\Delta}=M^{-1}-M_{0}^{-1}$. 
Define $\boldsymbol{x}_{1}(t)=\boldsymbol{q}(t)$ and $\boldsymbol{x}_{2}(t)=\dot{\boldsymbol{q}}(t)$, respectively. The dynamics (2) can be rewritten as

$$
\left\{\begin{array}{l}
\dot{\boldsymbol{x}}_{1}=\boldsymbol{x}_{2} \\
\dot{\boldsymbol{x}}_{2}=M_{0}^{-1} \boldsymbol{\tau}+M^{-1} \boldsymbol{d}+\boldsymbol{l}
\end{array}\right.
$$

Define the system tracking errors

$$
\left\{\begin{array}{l}
e_{1}=\boldsymbol{x}_{1}-\boldsymbol{x}_{d} \\
\boldsymbol{e}_{2}=\dot{\boldsymbol{x}}_{1}-\dot{\boldsymbol{x}}_{d}=\boldsymbol{x}_{2}-\dot{\boldsymbol{x}}_{d}
\end{array}\right.
$$

where $\boldsymbol{x}_{d}$ is the command trajectory, substituting (3) into (4), the error system is obtained as

$$
\left\{\begin{array}{l}
\dot{e}_{1}=e_{2} \\
\dot{e}_{2}=l+M_{0}^{-1} \boldsymbol{\tau}+M^{-1} \boldsymbol{d}-\ddot{\boldsymbol{x}}_{d}
\end{array}\right.
$$

Consider that the control input $\boldsymbol{\tau}=\left[\tau_{1}, \tau_{2}, \cdots, \tau_{n}\right]^{\mathrm{T}}$ is subjected to the following nonsymmetric constraints

$$
\tau_{L i} \leq \tau_{i} \leq \tau_{H i}, i=1,2, \cdots, n
$$

where $\tau_{L i}$ and $\tau_{H i}$ are the known lower and upper limitations of actuator outputs. Thus, the control input $\tau_{i}$ is defined by

$$
\tau_{i}= \begin{cases}\tau_{H i} & \text { if } \tau_{i} \geq \tau_{H i} \\ \tau_{0 i} & \text { if } \tau_{L i} \leq \tau<\tau_{H i} \\ \tau_{L i} & \text { if } \tau_{i}<\tau_{L i}\end{cases}
$$

The control input can be expressed as

$$
\boldsymbol{\tau}(t)=\boldsymbol{\tau}_{0}(t)+\boldsymbol{\tau}_{\Delta}(t)
$$

where $\tau_{0}=\left[\tau_{01}, \tau_{02}, \cdots, \tau_{0 n}\right]^{\mathrm{T}}$ is the nominal design part, the unknown part $\tau_{\Delta}=\left[\tau_{\Delta 1}, \tau_{\Delta 2}, \cdots, \tau_{\Delta n}\right]^{\mathrm{T}}$ is given as

$$
\tau_{\Delta i}=\left\{\begin{array}{ll}
\tau_{H i}-\tau_{0 i} & \text { if } \tau_{0 i} \geq \tau_{H i} \\
0 & \text { if } \tau_{L i} \leq \tau_{0 i}<\tau_{H i} \\
\tau_{L i}-\tau_{0 i} & \text { if } \tau_{0 i}<\tau_{L i}
\end{array} \quad i=1,2, \ldots, n\right.
$$

Before the statement of the control objective of this study, following assumptions are claimed.

Assumption 1: The unknown part of the control input is bounded by $\left\|\tau_{\Delta i}\right\| \leq \bar{\tau}_{\Delta}$, where $\bar{\tau}_{\Delta}$ is a known constant.

Assumption 2: The states $\boldsymbol{x}_{1}(t)$ and $\boldsymbol{x}_{2}(t)$ are measured precisely in real time by the sensors mounted on the robotic manipulators. The desired trajectory of the robotic manipulators $\boldsymbol{x}_{d}(t)$ and its time derivatives $\dot{\boldsymbol{x}}_{d}(t), \ddot{\boldsymbol{x}}_{d}(t)$ are known and bounded.

Assumption 3: [35] [36] The modeling uncertainty $\boldsymbol{l}(t)$ is unknown but bounded, the external disturbances are unknown but bounded by $\left\|M^{-1} \boldsymbol{d}\right\| \leq \bar{d}$, where $\bar{d}$ is a known constant.

Remark 1: The modeling uncertainty $\boldsymbol{l}(t)$ is a statedependent function. Especially in this study, $\boldsymbol{l}(t)$ depends on the position $\boldsymbol{q}$, the velocity $\dot{\boldsymbol{q}}$ and the torque input $\boldsymbol{\tau}$ of the robot joint. Since the input power of the mechanical equipment is limited by electric energy and the robot's motion space is limited and continuous, then the modeling uncertainty $l(t)$ can be assumed bounded.

The goal of this study is to design a controller so that the motion trajectory $\boldsymbol{x}_{1}(t)$ of the robot manipulator follows the desired trajectory $\boldsymbol{x}_{d}(t)$ to a small bounded set, and find the best control scheme to reduce the control cost, while all signals in the closed-loop system remain bounded. The block diagram of the proposed control system is shown in Fig. 1.

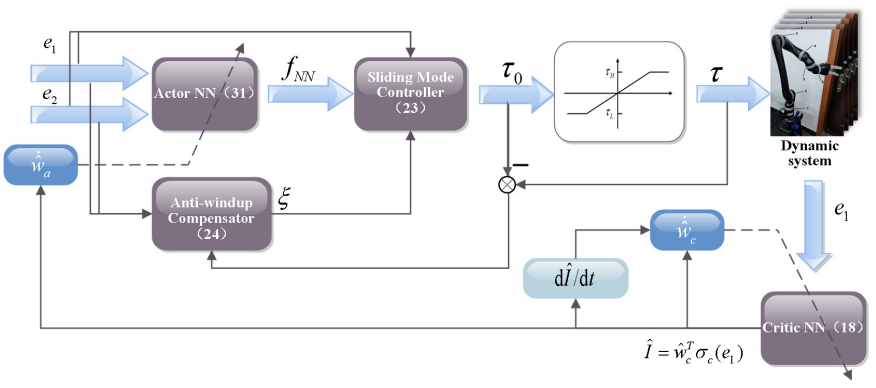

Fig. 1: The block diagram of the proposed control system

\section{Controller Design and Stability Analysis}

\section{A. Preliminary}

Based on the approximation property of the neural networks, the continuous-time function $c(Z)$ can be represented as

$$
\boldsymbol{c}(Z)=\boldsymbol{w}^{* \mathrm{~T}} \boldsymbol{\sigma}(Z)+\varepsilon^{*}
$$

where $\boldsymbol{w}^{*}=\left[w_{1}^{*}, w_{2}^{*}, \ldots, w_{r}^{*}\right]^{\mathrm{T}} \in \mathbb{R}^{r}$ denotes the optimal weight vector, $r>1$ represents the number of nodes in the hidden layer, $Z=\left[z_{1}, z_{2}, \ldots, z_{n}\right]^{\mathrm{T}} \in \Omega \subset \mathbb{R}^{n}$ is the input vector, and $\varepsilon^{*}$ is called the minimal approximation error, $\sigma(Z)$ employs gaussian function in the form of [37]

$$
\boldsymbol{\sigma}(Z)=\exp \left[\frac{-\left(Z-\mu_{i}\right)^{\mathrm{T}}\left(Z-\mu_{i}\right)}{\chi_{k}^{2}}\right], i=1,2, \ldots, r
$$

where $\mu_{i}=\left[\mu_{i 1}, \mu_{i 2}, \ldots, \mu_{i n}\right]$ is the centre of receptive field, $\chi_{k}$ is the width of the Gaussian function.

Lemma 1: [38] For $y_{i} \in \mathbb{R}, i=1,2, \cdots, n, 0<r_{1} \leq$ $1, r_{2}>1$, the following inequalities hold:

$$
\begin{gathered}
\left(\sum_{i=1}^{n}\left|y_{i}\right|\right)^{r_{1}} \leq \sum_{i=1}^{n}\left|y_{i}\right|^{r_{1}} \\
\left(\sum_{i=1}^{n}\left|y_{i}\right|\right)^{r_{2}} \leq n^{r_{2}-1} \sum_{i=1}^{n}\left|y_{i}\right|^{r_{2}}
\end{gathered}
$$

Lemma 2: [39] Consider the following nonlinear system $\dot{\boldsymbol{x}}=f(t, \boldsymbol{x}), \boldsymbol{x}(0)=\boldsymbol{x}_{0}$, where $\boldsymbol{x} \in \mathbb{R}^{n}, f: \mathbb{R}_{+} \times \mathbb{D} \rightarrow \mathbb{R}^{n}$ is a continuous function defined on an open neighborhood $G \in \mathbb{R}^{n}$ of the origin and satisfies $f(0)=0$. Suppose there exists a positive definite scalar function $V(\boldsymbol{x}): \mathbb{R}^{n} \rightarrow \mathbb{R}$ satisfying

(i) $V(\boldsymbol{x})=0 \Rightarrow \boldsymbol{x}=0$

(ii) $\dot{V}(\boldsymbol{x}) \leq-\left(\alpha V^{c}(\boldsymbol{x})+\beta V^{q}(\boldsymbol{x})\right)^{k}+\epsilon$

where $\alpha, \beta, c, q, k$ are positive parameters, $c k<1, q k>1$, the origin of system $\dot{\boldsymbol{x}}=f(t, \boldsymbol{x}), \boldsymbol{x}(0)=\boldsymbol{x}_{0}$ is practical fixedtime stable. Moreover, the system states converge to a region $\Omega$ in fixed time $T$, where

$$
\Omega=\left\{\boldsymbol{x} \mid\|\boldsymbol{x}\| \leq \min \left\{\frac{1}{\alpha^{k}}\left(\frac{\epsilon}{1-\theta}\right)^{\frac{1}{k c}}, \frac{1}{\beta^{k}}\left(\frac{\epsilon}{1-\theta}\right)^{\frac{1}{k q}}\right\}\right\}
$$

and the convergence time can be expressed as $T \leq$ $1 /\left(\alpha^{k} \theta^{k}(1-c k)\right)+1 /\left(\beta^{k} \theta^{k}(q k-1)\right), 0<\theta<1$. 


\section{B. Critic Neural Network Design for Reinforcement Learning}

A long-term cost function is given as [32]

$$
I(t)=\int_{t}^{\infty} \mathrm{e}^{-\frac{\iota-t}{\psi}} \varphi(\iota) \mathrm{d} \iota
$$

where $\psi$ is a constant used to discount the future cost; $\varphi(t)$ represents an instant cost function expressed as

$$
\varphi(t)=\left(\boldsymbol{x}_{1}-\boldsymbol{x}_{d}\right)^{\mathrm{T}} D\left(\boldsymbol{x}_{1}-\boldsymbol{x}_{d}\right)+\boldsymbol{\tau}^{\mathrm{T}} R \boldsymbol{\tau}
$$

where $D$ and $R$ represent positive definite matrices and $\boldsymbol{x}_{1}=$ $\boldsymbol{q}, \boldsymbol{x}_{d}=\boldsymbol{q}_{d}$ denotes the desired trajectory which is assumed to be continuous and known. The optimal control is realised when obtaining the minimum cost-to-go.

Define $I=\boldsymbol{w}_{c}^{* \mathrm{~T}} \boldsymbol{\sigma}_{c}\left(\boldsymbol{e}_{1}\right)+\varepsilon_{c}$ and $\hat{I}=\hat{\boldsymbol{w}}_{c}^{\mathrm{T}} \boldsymbol{\sigma}_{c}\left(\boldsymbol{e}_{1}\right)$. As describing the cost function (14), the approximation error of the cost-to-go function is expressed as

$$
\gamma(t)=\varphi(t)-\frac{1}{\psi} \hat{I}(t)+\dot{\hat{I}}(t)
$$

where $\psi$ is an adjustable constant. According to the gradient descent, the updating law of critic network is designed as [32]

$$
\dot{\hat{\boldsymbol{w}}}_{c}=-\delta_{c} \frac{\partial E_{c}}{\partial \boldsymbol{w}_{c}}
$$

where $E_{c}=\frac{1}{2} \gamma^{2}$. Substituting (15) into (16), one has

$$
\begin{aligned}
\dot{\hat{\boldsymbol{w}}}_{c} & =-\delta_{c} \gamma \frac{\partial \gamma}{\partial \hat{\boldsymbol{w}}_{c}} \\
& =-\delta_{c} \gamma \frac{\partial[\varphi(t)-(1 / \psi) \hat{I}(t)+\dot{\hat{I}}(t)]}{\partial \hat{\boldsymbol{w}}_{c}} \\
& =-\delta_{c} \gamma\left[-\frac{1}{\psi} \frac{\partial \hat{I}}{\partial \hat{\boldsymbol{w}}_{c}}+\frac{\partial}{\partial \hat{\boldsymbol{w}}_{c}}\left(\frac{\partial \hat{I}}{\partial \boldsymbol{e}_{1}}\right) \dot{\boldsymbol{e}}_{1}\right] \\
& =-\delta_{c}\left(\varphi(t)+\hat{\boldsymbol{w}}_{c}^{\mathrm{T}} \Lambda\right) \Lambda=-\delta_{c} \boldsymbol{\rho}_{c}\left(\hat{\boldsymbol{w}}_{c}, \boldsymbol{e}_{1}, \dot{\boldsymbol{e}}_{1}\right)
\end{aligned}
$$

where the constant $\delta_{c}>0$ is the learning rate of the critic $\mathrm{NN}$, and $\Lambda=-\left(\boldsymbol{\sigma}_{c} / \psi\right)+\nabla \boldsymbol{\sigma}_{c} \dot{\boldsymbol{e}}_{1}$. Parameters projection is applied to ensure boundedness of weight vector of critic NN. A constant vector $\overline{\boldsymbol{w}}_{c}=\left[\bar{w}_{c 1}, \bar{w}_{c 2}, \cdots, \bar{w}_{c r}\right]^{\mathrm{T}}$ is chosen and satisfied $\left|\hat{w}_{c i}\right| \leq \bar{w}_{c i}(i=1,2, \cdots, l)$. The updating law based on projection method for critic $\mathrm{NN}$ is designed as

$$
\dot{\hat{\boldsymbol{w}}}_{c}= \begin{cases}-\delta_{c} \boldsymbol{\rho}_{c} & \left\|\hat{\boldsymbol{w}}_{c}\right\| \leq\left\|\overline{\boldsymbol{w}}_{c}\right\| \text { or } \\ & \left\|\hat{\boldsymbol{w}}_{c}\right\|=\left\|\overline{\boldsymbol{w}}_{c}\right\|, \boldsymbol{w}_{c}^{\mathrm{T}} \boldsymbol{\rho}_{c}>0 \\ -\delta_{c} \boldsymbol{\rho}_{c}+\delta_{c} \boldsymbol{\xi}_{c} & \left\|\hat{\boldsymbol{w}}_{c}\right\|=\left\|\overline{\boldsymbol{w}}_{c}\right\|, \boldsymbol{w}_{c}^{\mathrm{T}} \boldsymbol{\rho}_{c} \leq 0\end{cases}
$$

where $\boldsymbol{\xi}_{c}=\frac{\hat{\boldsymbol{w}}_{c}^{\mathrm{T}} \boldsymbol{\rho}_{c}}{\left\|\hat{\boldsymbol{w}}_{c}\right\|^{2}} \hat{\boldsymbol{w}}_{c}, \boldsymbol{\rho}_{c}$ and $\boldsymbol{\xi}_{c}$ are bounded.

Remark 2: The approximate error of the cost-to-go function (15) is expressed in terms of the derivative of $I(t)$ in order to use the state $\dot{e}_{1}$. After adding $\dot{e}_{1}$ as input, the neural network approximates the function $I$ more accurately.

\section{Actor Neural Network-Based Controller Design}

In this section, a reinforcement learning fixed-time nonsingular terminal sliding mode(RL-FTNTSM) controller is designed to achieve the tracking objective. In order to guarantee that $e_{1}$ and $e_{2}$ can converge to small neighborhoods around zero in a uniformly bounded convergence time $T_{s}$, where $T_{s}$ is independent with the initial states, a novel non-singular fixedtime fast terminal sliding mode is adopted as

$$
\boldsymbol{s}=K\left(\boldsymbol{e}_{1}\right) \boldsymbol{e}_{1}+\operatorname{sig}^{v_{1}}\left(\boldsymbol{e}_{2}\right)
$$

where $K\left(\boldsymbol{e}_{1}\right)=\operatorname{diag}\left\{k_{e_{11}}, k_{e_{12}}, \cdots, k_{e_{1 n}}\right\}$ is a diagonal matrix and $k_{e_{1 i}}, i=1,2, \cdots, n$ are denoted as

$$
k_{e_{1 i}}=\left(\alpha\left|e_{1 i}\right|^{p-1 /\left(k v_{1}\right)}+\beta\left|e_{1 i}\right|^{g-1 /\left(k v_{1}\right)}\right)^{k v_{1}}
$$

where $\alpha>0, \beta>0, k>1, v_{1}>1$ and $p, g$ are positive scalars satisfying that $g k>1$ and $1 / v_{1}<p k<1$.

Remark 3: The coefficient of the sliding mode surface $K\left(\boldsymbol{e}_{1}\right)$ is time-varying related to the error $\boldsymbol{e}_{1}$. Compared with the traditional non-singular terminal sliding mode, the sliding mode (19) can quickly pull the system state back to the sliding mode surface when the error is large. It can be seen from the results in [40] that the sliding mode (19) has faster convergence performance compared with other traditional terminal sliding modes.

The time derivative of $s$ is

$$
\dot{\boldsymbol{s}}=\tilde{K}\left(\boldsymbol{e}_{1}\right) \boldsymbol{e}_{2}+K\left(\boldsymbol{e}_{1}\right) \boldsymbol{e}_{2}+v_{1} E_{2} \dot{\boldsymbol{e}}_{2}
$$

where $E_{2}=\operatorname{diag}\left\{\left|e_{21}\right|^{v_{1}-1},\left|e_{22}\right|^{v_{1}-1}, \cdots,\left|e_{2 n}\right|^{v_{1}-1}\right\}$, $\tilde{K}\left(\boldsymbol{e}_{1}\right)=\operatorname{diag}\left\{\tilde{k}_{e_{11}}, \tilde{k}_{e_{12}}, \cdots, \tilde{k}_{e_{1 n}}\right\}$ is a diagonal matrix and $\tilde{k}_{e_{1 i}}, i=1,2, \cdots, n$ are denoted as

$$
\begin{aligned}
\tilde{k}_{e_{1 i}}= & k v_{1}\left(\alpha\left|e_{1 i}\right|^{p-1 / k v_{1}}+\beta\left|e_{1 i}\right|^{g-1 / k v_{1}}\right)^{k v_{1}-1} \\
& \left\{\alpha\left(p-\frac{1}{k v_{1}}\right)\left|e_{1 i}\right|^{p-1 / k v_{1}}+\beta\left(g-\frac{1}{k v_{1}}\right)\left|e_{1 i}\right|^{g-1 / k v_{1}}\right\}
\end{aligned}
$$

Remark 4: It should be noted that $\tilde{K}\left(\boldsymbol{e}_{1}\right)$ is not the derivative of $K\left(\boldsymbol{e}_{1}\right)$.

The modeling uncertainty $\boldsymbol{l}$ in (5) is unknown, so an actor NN is adopted to estimate the uncertainty. Thus, the RLFTNTSM controller is designed as

$$
\begin{aligned}
\boldsymbol{\tau}_{0}= & M_{0}\left[-\frac{1}{v_{1}}\left(\tilde{K}\left(\boldsymbol{e}_{1}\right)+K\left(\boldsymbol{e}_{1}\right)\right) \operatorname{sig}^{2-v_{1}}\left(\boldsymbol{e}_{2}\right)\right. \\
& -\frac{1}{v_{1}}\left(\operatorname{sig}^{1-v_{1}}\left(\boldsymbol{e}_{2}\right)\right)\left(\operatorname{sig}^{v_{4}}\left(\sigma_{1} \operatorname{sig}^{v_{2}}(\boldsymbol{s})+\sigma_{2} \operatorname{sig}^{v_{3}}(\boldsymbol{s})\right)\right. \\
& \left.\left.+\boldsymbol{\zeta}+K_{s} \boldsymbol{s}\right)+\ddot{\boldsymbol{x}}_{d}-\boldsymbol{f}_{N N}-\bar{d} \operatorname{sign}(\boldsymbol{s})\right]
\end{aligned}
$$

where $v_{4}>1, v_{2} v_{4}<1$, and $1<v_{3} v_{4}<v_{4} . \sigma_{1}$ and $\sigma_{2}$ are positive constants. $K_{s}=\operatorname{diag}\left\{k_{s 1}, k_{s 2}, \cdots, k_{s n}\right\}$ is a diagonal positive definite matrix with $k_{s i}>0, i=1,2, \cdots, n$. The anti-windup compensator can be expressed as

$$
\dot{\boldsymbol{\zeta}}=-K_{\zeta} \boldsymbol{\zeta}-\operatorname{sig}^{v_{4}}\left(\sigma_{3} \operatorname{sig}^{v_{2}}(\boldsymbol{\zeta})+\sigma_{4} \operatorname{sig}^{v_{3}}(\boldsymbol{\zeta})\right)+\boldsymbol{s}+\boldsymbol{\tau}_{\Delta}
$$

where $K_{\zeta}$ is positive definite matrices; $\sigma_{3}$ and $\sigma_{4}$ are positive constants.

The $\boldsymbol{f}_{N N}$ is an output vector of RBFNN that approximate the modeling uncertainty $l$ which is expressed as

$$
\boldsymbol{f}_{N N}=\left[\begin{array}{c}
\hat{\boldsymbol{w}}_{a 1}^{\mathrm{T}} \boldsymbol{\sigma}_{a 1}\left(\boldsymbol{z}_{a 1}\right) \\
\hat{\boldsymbol{w}}_{a 2}^{\mathrm{T}} \boldsymbol{\sigma}_{a 2}\left(\boldsymbol{z}_{a 2}\right) \\
\cdots \\
\hat{\boldsymbol{w}}_{a n}^{\mathrm{T}} \boldsymbol{\sigma}_{a n}\left(\boldsymbol{z}_{a n}\right)
\end{array}\right]
$$

where the $\boldsymbol{w}_{a i}=\left[w_{a i 1}, w_{a i 2}, \cdots, w_{a i m}\right]^{\mathrm{T}}$ is the weight vector, $\boldsymbol{z}_{a i}=\left[e_{1 i}, e_{2 i}\right]^{\mathrm{T}}$ is the input vector of RBFNN, and 
$\boldsymbol{\sigma}_{a i}\left(\boldsymbol{z}_{a i}\right)$ is the guassian function. The learning error can be defined as

$$
\mu_{a}=\sum_{i=1}^{n} \tilde{\boldsymbol{w}}_{a i}^{\mathrm{T}} \boldsymbol{\sigma}_{a i}\left(\boldsymbol{z}_{a i}\right)
$$

where $\tilde{\boldsymbol{w}}_{a i}=\boldsymbol{w}_{a i}^{*}-\hat{\boldsymbol{w}}_{a i}$. Then the error with the actor $\mathrm{NN}$ is designed as

$$
e_{a}=\mu_{a}+k_{I}\left(\hat{I}(t)-I_{d}(t)\right)
$$

where $I_{d}=0$ denote the desired ideal cost-to-go in the future and $k_{I}$ represents a positive constant. Define

$$
E_{a}=\ln \left(\cosh e_{a}\right)
$$

The updating law of actor NN is designed as

$$
\begin{aligned}
\dot{\hat{\boldsymbol{w}}}_{a i} & =-\delta_{a} \frac{\partial E_{a}}{\partial \boldsymbol{w}_{a}} \\
& =-\delta_{a} \frac{\mathrm{d} E_{a}}{\mathrm{~d} e_{a}} \frac{\partial e_{a}}{\partial \mu_{a}} \frac{\partial \mu_{a}}{\partial \hat{\boldsymbol{w}}_{a i}} \\
& =-\delta_{a} \tanh \left(\mu_{a}+k_{I} \hat{I}\right) \boldsymbol{\sigma}_{a i}
\end{aligned}
$$

where $\delta_{a}>0$ is the learning rate for the actor NN. However, $\mu_{a}$ is unavailable, the modified updating law of the actor NN is designed as

$$
\begin{aligned}
\dot{\hat{\boldsymbol{w}}}_{a i} & =-\delta_{a} \tanh \left(\sum_{i=1}^{n} \hat{\boldsymbol{w}}_{a i}^{\mathrm{T}} \boldsymbol{\sigma}_{a i}+k_{I} \hat{I}\right) \boldsymbol{\sigma}_{a i} \\
& =-\delta_{a} \boldsymbol{\rho}_{a i}\left(\hat{\boldsymbol{w}}_{a i}, \boldsymbol{z}_{a i}\right)
\end{aligned}
$$

Parameters projection is applied to ensure boundedness of weight vector of actor NN. A class of constant vectors $\overline{\boldsymbol{w}}_{a i}=\left[\bar{w}_{a i 1}, \bar{w}_{a i 2}, \cdots, \bar{w}_{a i r}\right]^{\mathrm{T}}, i=1,2, \cdots, n$ are designed and satisfied $\left|\hat{w}_{a i j}\right| \leq \bar{w}_{a i j}(j=1,2, \cdots, r)$. The projection based updating law of actor NN is designed as

$$
\dot{\hat{\boldsymbol{w}}}_{a i}= \begin{cases}-\delta_{a} \boldsymbol{\rho}_{a i} & \left\|\hat{\boldsymbol{w}}_{a i}\right\| \leq\left\|\overline{\boldsymbol{w}}_{a i}\right\| \text { or } \\ & \left\|\hat{\boldsymbol{w}}_{a i}\right\|=\left\|\overline{\boldsymbol{w}}_{a i}\right\|, \boldsymbol{w}_{a i}^{\mathrm{T}} \boldsymbol{\rho}_{a i}>0 \\ -\delta_{a} \boldsymbol{\rho}_{a i}+\delta_{a} \boldsymbol{\xi}_{a i} & \left\|\hat{\boldsymbol{w}}_{a i}\right\|=\left\|\overline{\boldsymbol{w}}_{a i}\right\|, \boldsymbol{w}_{a i}^{\mathrm{T}} \boldsymbol{\rho}_{a i} \leq 0\end{cases}
$$

where $\boldsymbol{\xi}_{a i}=\frac{\hat{\boldsymbol{w}}_{a i}^{\mathrm{T}} \boldsymbol{\rho}_{a i}}{\left\|\hat{\boldsymbol{w}}_{a i}\right\|^{2}} \hat{\boldsymbol{w}}_{a i}, \boldsymbol{\rho}_{a i}$ and $\boldsymbol{\xi}_{a i}$ are bounded.

Remark 5: The equations (18) and (31) limit the weight vector of the neural network to a bounded range, which will provide a basis for the fixed-time stability analysis in the Appendix.

Theorem 1: Consider the manipulator with the proposed NN control, since the initial conditions are bounded, the closedloop system signals $\hat{\boldsymbol{w}}_{c}$ and $\hat{\boldsymbol{w}}_{a}$ are bounded and their initial values are set as $\left\|\hat{\boldsymbol{w}}_{c}\right\|=0$ and $\left\|\hat{\boldsymbol{w}}_{a}\right\|=0$. By using parametric projections (18) and (31), the boundedness of the weight vectors of critic NN and actor NN is guaranteed.

Proof: In order to prove the boundedness of parameter $\hat{\boldsymbol{w}}_{c}$, a candidate Lyapunov function is selected as

$$
V_{c}(t)=\frac{1}{2 \delta_{c}} \hat{\boldsymbol{w}}_{c}^{\mathrm{T}} \hat{\boldsymbol{w}}_{c}
$$

According to the projection updating law (18), there exists two cases to consider:

Case 1: If $\left\|\hat{\boldsymbol{w}}_{c}\right\| \leq\left\|\overline{\boldsymbol{w}}_{c}\right\|$ or $\left\|\hat{\boldsymbol{w}}_{c}\right\|=\left\|\overline{\boldsymbol{w}}_{c}\right\|, \hat{\boldsymbol{w}}_{c}^{\mathrm{T}} \boldsymbol{\rho}_{c}>0$, one has

$$
\dot{V}_{c}(t)=\frac{1}{\delta_{c}} \hat{\boldsymbol{w}}_{c}^{\mathrm{T}} \dot{\boldsymbol{\boldsymbol { w }}}_{c}=-\hat{\boldsymbol{w}}_{c}^{\mathrm{T}} \boldsymbol{\rho}_{c}<0
$$

Case 2: If $\left\|\hat{\boldsymbol{w}}_{c}\right\|=\left\|\overline{\boldsymbol{w}}_{c}\right\|, \hat{\boldsymbol{w}}_{c}^{\mathrm{T}} \boldsymbol{\rho}_{c} \leq 0$, one has

$$
\dot{V}_{c}(t)=\frac{1}{\delta_{c}} \hat{\boldsymbol{w}}_{c}^{\mathrm{T}} \dot{\hat{\boldsymbol{w}}}_{c}=-\hat{\boldsymbol{w}}_{c}^{\mathrm{T}} \boldsymbol{\rho}_{c}+\frac{\hat{\boldsymbol{w}}_{c}^{\mathrm{T}} \boldsymbol{\rho}_{c}}{\left\|\hat{\boldsymbol{w}}_{c}\right\|^{2}} \hat{\boldsymbol{w}}_{c}^{\mathrm{T}} \hat{\boldsymbol{w}}_{c}=0
$$

As long as $\left\|\hat{\boldsymbol{w}}_{c}(0)\right\| \leq\left\|\overline{\boldsymbol{w}}_{c}\right\|$ is satisfied, the projection updating law (18) can always ensure $\left\|\hat{\boldsymbol{w}}_{c}\right\| \leq\left\|\overline{\boldsymbol{w}}_{c}\right\|$. Similarly, a candidate Lyapunov function is selected for actor NN as

$$
V_{a}(t)=\frac{1}{2 \delta_{a}} \sum_{i=1}^{n} \hat{\boldsymbol{w}}_{a i}^{\mathrm{T}} \hat{\boldsymbol{w}}_{a i}
$$

Using the same method, the projection updating law (31) can always ensure $\left\|\hat{\boldsymbol{w}}_{a i}\right\| \leq\left\|\overline{\boldsymbol{w}}_{a i}\right\|, i=1,2, \cdots, n$. The proof is completed.

Theorem 2: Consider the manipulator system (1) under Assumptions 1-2, and the controller (23) with the adaptation laws (17) (30) and (24), it is true that by choosing appropriate parameters, the expected trajectory can be tracked in a fixed time, and the tracking errors $\boldsymbol{e}_{1}$ and $\boldsymbol{e}_{2}$ converge to small neighborhoods around zero. In addition, the upper bound of the convergent time can be estimated by

$$
T<T_{\max }=T_{1}+T_{2}
$$

where $T_{1}=\frac{2}{(3 n+1)^{1-v_{4}} \sigma_{5}^{v_{4}} \theta^{v_{4}}\left(1-v_{2} v_{4}\right)}+\frac{2}{(3 n+1)^{1-v_{4}} \sigma_{6}^{v_{4}} \theta^{v_{4}\left(v_{3} v_{4}-1\right)}}$, $\sigma_{5}=2^{\left(1+v_{2} v_{4}\right) / 2 v_{4}} \min \left\{\sigma_{1}, \sigma_{3}, 1\right\}, \sigma_{6}=$ $2^{\left(1+v_{3} v_{4}\right) / 2 v_{4}} \min \left\{\sigma_{2}, \sigma_{4}, 1\right\} ; T_{2}=\frac{1}{\alpha^{k} \theta^{k}(1-p k)}+\frac{1}{\beta^{k} \theta^{k}(g k-1)}$.

Proof: The proof of Theorem 2 is included in the Appendix.

Remark 6: The parameters in the article seem to be a lot, but in fact, it just needs to find a set of parameters within the specified range and fine-tune them. It should be noted that $\alpha$ and $\beta$ are key parameters, and the convergence time of the state can be adjusted by changing $\alpha$ and $\beta$. Reducing $p, g$ and $k$ can shorten the convergence time, but larger chattering will be generated and the steady-state accuracy will be reduced. If the value of $v_{1}$ is too large, the torque intput will change dramatically. Similarly, if the values of $v_{2}, v_{3}$ and $v_{4}$ are too small, then torque intput will chattering violently. Excessive values of $\sigma_{1}, \sigma_{2}, \sigma_{3}$ and $\sigma_{4}$ lead to greater chattering in both the state trajectory and the torque input. $K_{s}$ and $K_{\zeta}$ can be set to a larger constant matrix, but overlarge control gain will excite high-frequency dynamics of the robot system. The value of $\bar{d}$ should be greater than the amplitude of external disturbance.

\section{Simulations And Experiments}

\section{A. Numerical Simulations and Comparisons}

A two-link rigid planar robotic manipulator is used in simulations. The physical parameters are derived from [5]. Define $\boldsymbol{x}_{1}=\left[x_{11}, x_{12}\right]^{\mathrm{T}}$ as joint angles of the robotic manipulator. Then, the related matrices in the dynamics (1) for two-link rigid robotic manipulators are

$$
\begin{gathered}
M(\boldsymbol{x})=\left[\begin{array}{cc}
p_{1}+p_{2}+2 p_{3} \cos x_{12} & p_{2}+p_{3} \cos x_{12} \\
p_{2}+p_{3} \cos x_{12} & p_{2}
\end{array}\right] \\
C(\boldsymbol{x}, \dot{\boldsymbol{x}})=\left[\begin{array}{cc}
-p_{3} \dot{x}_{2} \sin x_{12} & -p_{3}\left(\dot{x}_{11}+\dot{x}_{12}\right) \sin x_{12} \\
p_{3} \dot{x}_{11} \sin x_{12} & 0
\end{array}\right]
\end{gathered}
$$




$$
\boldsymbol{g}(\boldsymbol{x})=\left[\begin{array}{c}
p_{4} g \cos x_{11}+p_{5} g \cos \left(x_{11}+x_{12}\right) \\
p_{5} g \cos \left(x_{11}+x_{12}\right)
\end{array}\right]
$$

where $p_{1}=m_{1} l_{c 1}^{2}+m_{2} l_{1}^{2}+I_{1}, p_{2}=m_{2} l_{c 2}^{2}+I_{2}, p_{3}=$ $m_{2} l_{1} l_{c 2}, p_{4}=m_{1} l_{c 2}+m_{2} l_{1}, p_{5}=m_{2} l_{c 2} ; m_{i}$ and $l_{i}$ are the mass and length of link $i ; m_{1}=2.00(\mathrm{~kg}), m_{2}=0.85(\mathrm{~kg})$, $l_{1}=0.35(\mathrm{~m}), l_{2}=0.31(\mathrm{~m}) ; I_{i}$ is the moment of inertia of link $i, I_{1}=\frac{1}{4} m_{1} l_{1}^{2}\left(\mathrm{kgm}^{2}\right), I_{2}=\frac{1}{4} m_{2} l_{2}^{2}\left(\mathrm{kgm}^{2}\right) ; l_{c i}$ is the distance between joint $i-1$ and the center of mass of link $i ; g=9.8\left(\mathrm{~m} / \mathrm{s}^{2}\right)$. The disturbance torque is $\boldsymbol{d}(t)=$ $[0.5(\sin (0.1 t)+1), 0.5(\cos (0.1 t)+1)]^{\mathrm{T}}(\mathrm{Nm})$.

The initial velocity of the robotic manipulators is $x_{21}(0)=$ $x_{22}(0)=0(\mathrm{rad} / \mathrm{s})$, the weight vector of $\mathrm{NN}$ is set as zero vector. The desired trajectory is $\boldsymbol{x}_{d}=[0.1(\sin (0.5 t)+$ $\cos (0.5 t), 0.1 \sin (t)+\cos (t)]^{\mathrm{T}}(\mathrm{rad})$, where $t \in\left[0, t_{m}\right]$ and $t_{m}=50(\mathrm{~s})$.

To verify the effectiveness of the proposed control scheme, a comparing simulation of two different control strategies are conducted here. The two different control schemes are the proposed saturated fixed-time trajectory tracking based on reinforcement learning controller (23) and the robust extended state observer (ESO) based NTSM controller in [5]. The controller proposed in [5] is expressed as

$$
\begin{aligned}
\boldsymbol{\tau}= & -M_{0}\left[\boldsymbol{z}_{3}+\frac{\sigma}{\gamma} \operatorname{sig}^{2-\gamma}\left(\boldsymbol{\delta}_{2}\right) K_{1} \frac{\gamma}{\sigma}\left\lfloor\boldsymbol{\delta}_{2}\right\rceil^{\gamma-1} \boldsymbol{s}\right. \\
& \left.+K_{2} \tanh \left(\boldsymbol{s} / \rho^{2}\right)-\ddot{\boldsymbol{x}}_{d}\right]
\end{aligned}
$$

where $M_{0}=\left[\cos x_{12}, \cos x_{12} ; \cos x_{12}, 1\right], z_{3}$ is the output vector of the extended state observer to compensate the compound disturbance $l$. The extended state observer is proposed to estimate the model uncertainties as

$$
\left\{\begin{array}{l}
\dot{z}_{1}=\boldsymbol{z}_{2}-\boldsymbol{\beta}_{1} \operatorname{sig}^{\kappa}\left(\boldsymbol{e}_{1}\right) \\
\dot{\boldsymbol{z}}_{\mathbf{2}}=\boldsymbol{z}_{3}-\boldsymbol{\beta}_{2} \operatorname{sig}^{\kappa}\left(\boldsymbol{e}_{1}\right)+M_{0}^{-1} \boldsymbol{\tau} \\
\dot{z}_{\mathbf{3}}=-\boldsymbol{\beta}_{3} \operatorname{sig}^{\kappa}\left(\boldsymbol{e}_{1}\right)
\end{array}\right.
$$

where $\kappa=\left(\alpha_{0}+1\right) / 2$ and $0<\alpha_{0}<1$ is an adjustable parameter; $\boldsymbol{\beta}_{i}$ is the observer gain. The adjustable parameters of ESO are selected as $\alpha_{0}=0.9, \boldsymbol{\beta}_{1}=100, \boldsymbol{\beta}_{2}=100$ and $\boldsymbol{\beta}_{3}=7400$. The parameters of NTSM are set as $\sigma=3$, $p=11, q=7, K_{1}=\operatorname{diag}\{10,10\}, K_{2}=\operatorname{diag}\{10,10\}$ and $\rho=10$. In controller (23), the adjustable parameters are designed as $\alpha=1.5, \beta=1.5, p=0.7, g=\frac{10}{9}, k=1.2$, $v_{1}=1.2 . v_{2}=\frac{1}{3}, v_{3}=\frac{3}{4}, v_{4}=2, \sigma_{1}=\sqrt{2}, \sigma_{2}=\sqrt{2}$, $K_{s}=\operatorname{diag}\{100,100\} . K_{\zeta}=\operatorname{diag}\{1,1\}, \sigma_{3}=\sqrt{2}, \sigma_{4}=\sqrt{2}$, $\bar{d}=2.2$. The number of Gaussian nodes for both actor and critic is $r=4$. The limitations of the actuator outputs are set as $\tau_{L i}=-5, \tau_{H i}=5,(i=1,2)$.

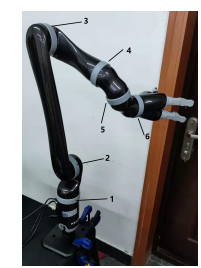

Fig. 2: Kinova JACO2
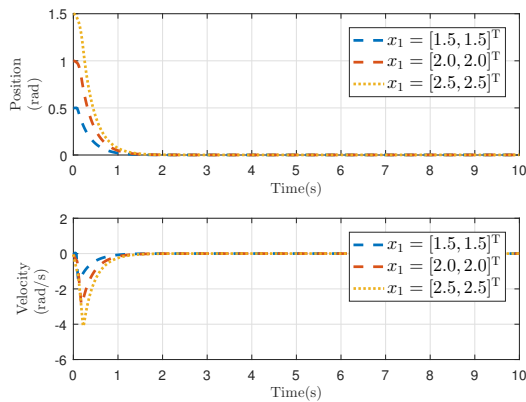

Fig. 3: Joint 1 tracking error by (23)
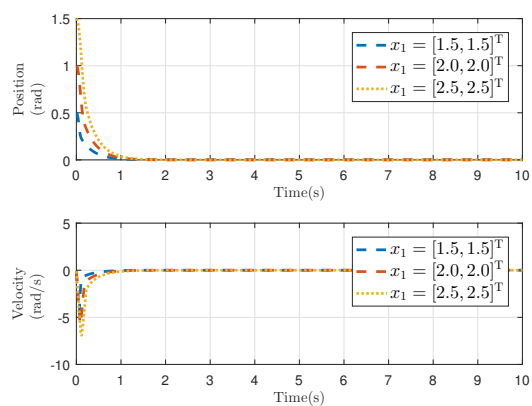

Fig. 4: Joint 2 tracking error by (23)
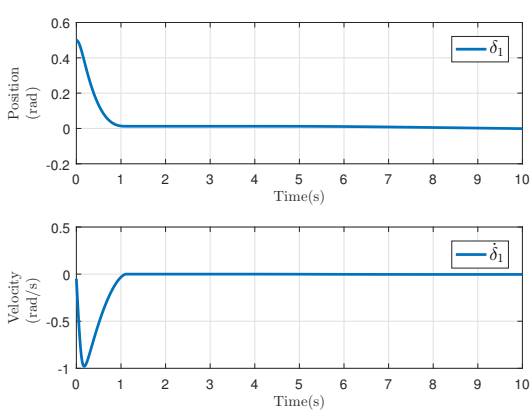

Fig. 5: Joint 1 tracking error by (36)
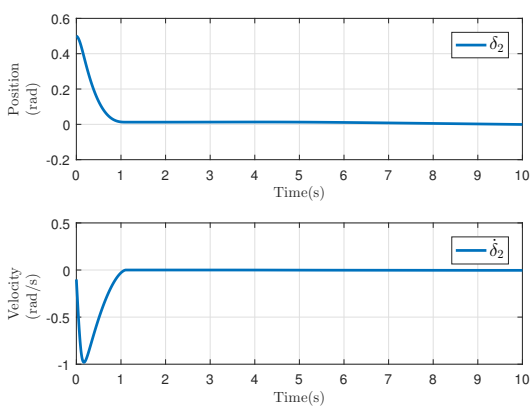

Fig. 6: Joint 2 tracking error by (36) 

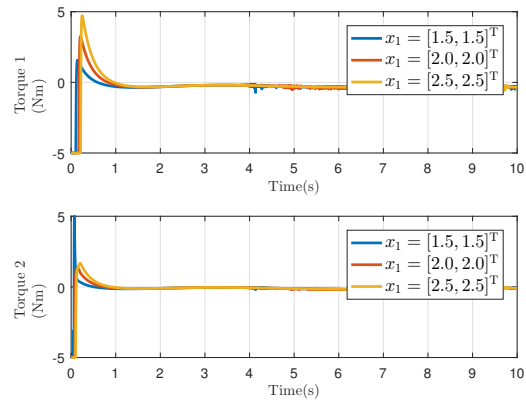

Fig. 7: Control input by (23)
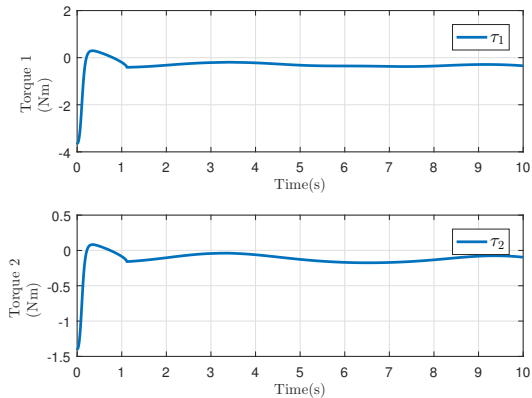

Fig. 8: Control input by (36)
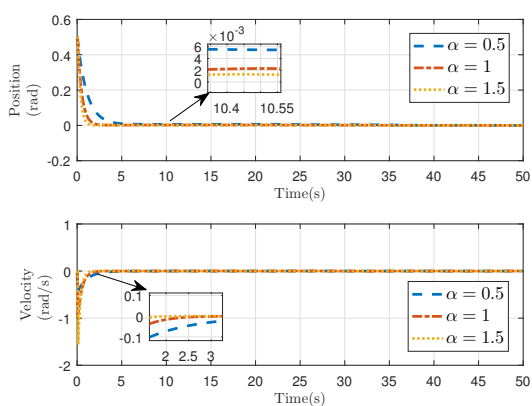

Fig. 9: Joint 1 tracking error under different $\alpha$ by (23)
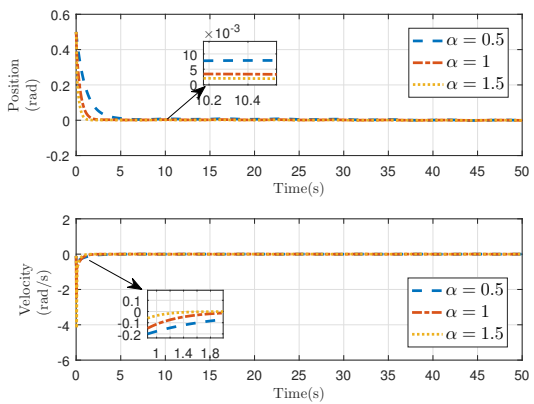

Fig. 10: Joint 2 tracking error under different $\alpha$ by (23)

As shown in Figs. 3 and 4, under different initial position conditions, the proposed controller can achieve satisfactory performances in finite time in spite of external disturbances and parametric uncertainties in model. Comparing Figs. 3 and 4 with Figs. 5 and 6, the controller proposed in this paper has a shorter convergence time and smoother transient fluctuation than the ones by using (36). The curve of control torque is shown in Figs. 7 and 8. During the steady-state phase, the actuator's output torque vibrates slightly. It can be seen from the convergence time estimation formula $T_{2}$ in Appendix that the two parameters $\alpha$ and $\beta$ have a relatively large impact on the convergence time, $\alpha$ is taken as an example for debugging, as shown in Figs. 9 and 10, the parameter $\alpha$ affects the convergence time, and the smaller $\alpha$, the longer the convergence time. Furthermore, in order to compare the performance of two controllers quantitatively, under the same initial conditions, two performance metrics including the integrated absolute error(IAE, defined by $\left.\int_{0}^{t}\left|\boldsymbol{e}_{1}(\boldsymbol{\tau})\right|+\left|\boldsymbol{e}_{2}(\boldsymbol{\tau})\right| \mathrm{d} \boldsymbol{\tau}\right)$ and the integrated time absolute error (ITAE, defined by $\int_{0}^{t} \tau\left|e_{1}(\tau)\right|+$ $\left.\boldsymbol{\tau}\left|\boldsymbol{e}_{2}(\boldsymbol{\tau})\right| \mathrm{d} \boldsymbol{\tau}\right)$ are applied to make the comparisons, while the results of performance indexes are shown in Table 1.

TABLE I: Performance comparisons between two methods

\begin{tabular}{cccc}
\hline & Error(rad) & Controller(23) & Controller(36) \\
\hline IAE & $e_{1}$ of joint 1 & 0.1952 & 0.2688 \\
& $e_{1}$ of joint 2 & 0.1150 & 0.2671 \\
ITAE & $e_{1}$ of joint 1 & 0.1015 & 0.4177 \\
& $e_{1}$ of joint 2 & 0.0772 & 0.4187 \\
\hline
\end{tabular}
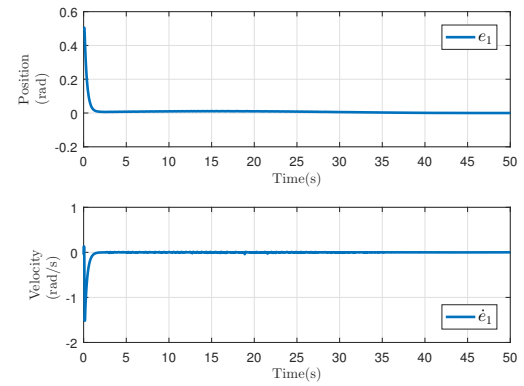

Fig. 11: Joint 1 tracking error by (23)
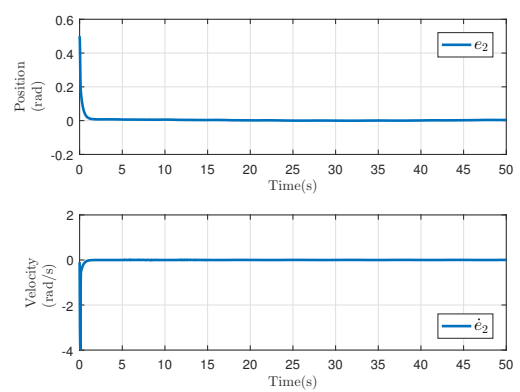

Fig. 12: Joint 2 tracking error by (23)

To verify the robust performance of the proposed control law, we increase the disturbance torque as $\boldsymbol{d}(t)=$ $[3(\sin (0.1 t)+1), 4(\cos (0.1 t)+1)]^{\mathrm{T}}(\mathrm{Nm})$. The simulation results are shown in Figs. 11-13, and it can be seen that the tracking errors of position and velocity still converge to a smaller neighborhood within a fixed time under the larger 
external disturbances. Since the initial weight value of the neural network is zero vector, it meets the requirement of Theorem 1. As can be seen from Fig. 14, the weight always remains bounded.
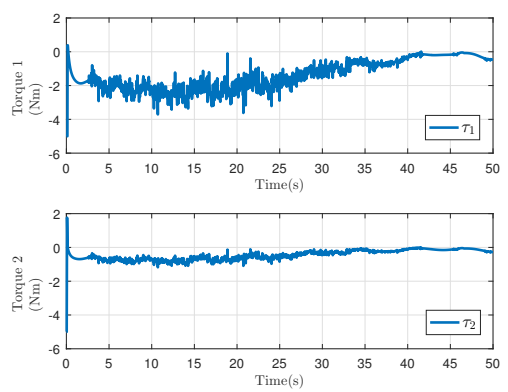

Fig. 13: Control input by (23)
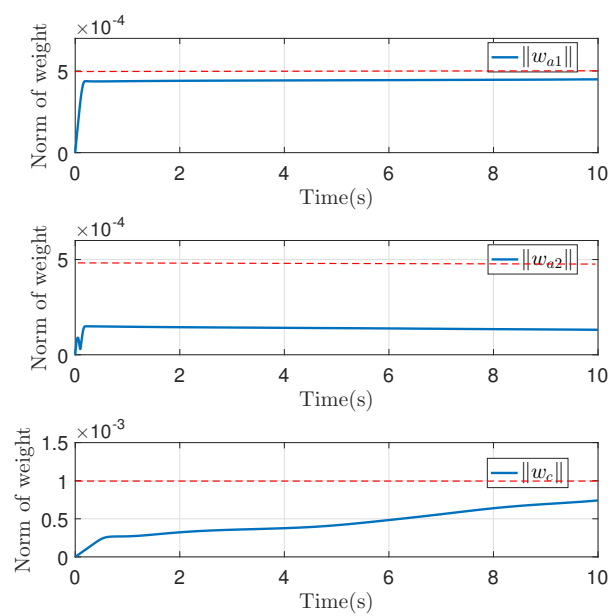

Fig. 14: Norm of weight

\section{B. Experimental Results and Performance Validations}

In order to further verify the superiority of the designed controller, based on Kinova JACO2, an experimental comparison was made between controller (23) and the controller proposed in [41]. The controller proposed in [41] is expressed as

$$
\begin{aligned}
\boldsymbol{\tau} & =-M_{0}\left[\boldsymbol{f}_{\mathrm{NN}}+\frac{\sigma}{\gamma} \operatorname{sig}^{2-\gamma}\left(\boldsymbol{\delta}_{2}\right)\right. \\
& \left.+K_{1} \frac{\gamma}{\sigma}\left\lfloor\boldsymbol{\delta}_{2}\right\rceil^{\gamma-1} \boldsymbol{s}+K_{2} \tanh \left(\boldsymbol{s} / \rho^{2}\right)-\ddot{\boldsymbol{x}}_{d}\right]
\end{aligned}
$$

Kinova JACO2 is a lightweight and portable six-degree-offreedom robotic arm as shown in Fig. 2. The manipulator can be directly controlled by Matlab, which facilitates rewriting the program from simulation to experiment. In the experiment, we first set the JACO2 as the torque control mode. In order to verify the effectiveness of the controller, two kinds of expected trajectories, exponential type and periodic type, are set for tracking, the third joint was selected to perform the experiments. After set JACO2 as the torque control mode, the manipulator has a certain degree of deflection due to gravity, but under the action of the controller, the actual trajectory can track the expected trajectory. The exponential expected trajectory is set as $x_{d}=2.3-\frac{7}{5} \mathrm{e}^{-0.5 t}+\frac{7}{20} \mathrm{e}^{-2 t}$, and the limitations of the actuator outputs are set as $\tau_{L i}=-2, \tau_{H i}=2$, $(i=1,2)$, Figs. 15 and 16 show the tracking errors and torque inputs of the two controllers for the exponential expected trajectory. It can be seen that the steady-state accuracy of the controller(23) proposed in this paper is better than that of the controller(38). The periodic expected trajectory is set as $x_{d}=1.3+0.3 \sin (t)$, and the limitations of the actuator outputs are set as $\tau_{L i}=-3, \tau_{H i}=3,(i=1,2)$. Figs. 17 and 18 show the tracking errors and torque inputs of the two controllers for the periodic expected trajectory, It can also be seen that the steady-state accuracy of the controller(23) proposed in this paper is better than that of the controller (38).
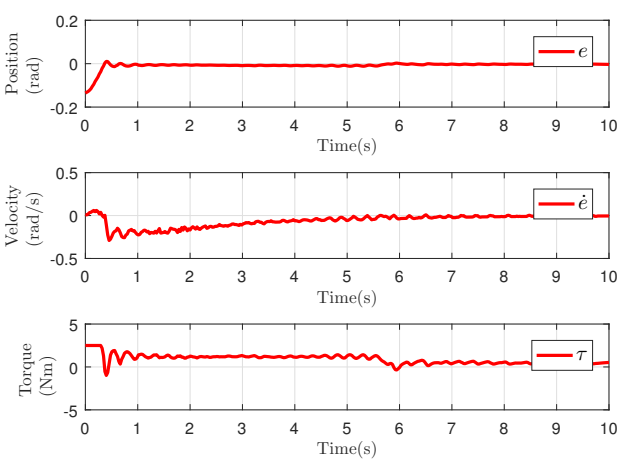

Fig. 15: Exponential tracking error and torque input by (23)
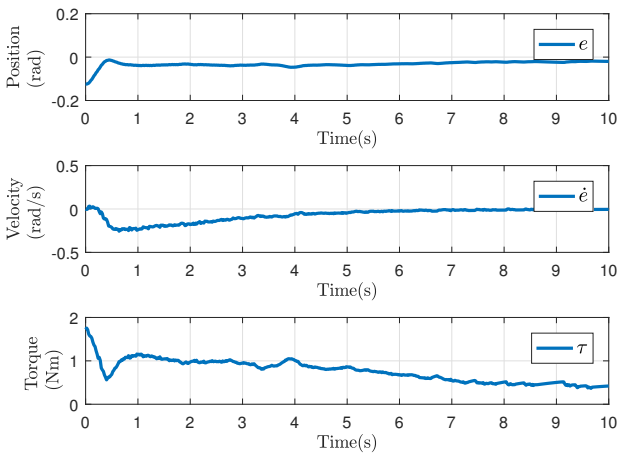

Fig. 16: Exponential tracking error and torque input by (38)

It can be seen from the experimental results that a large torque will be generated in the initial stage of the manipulator operation. At this time, the anti-saturation compensator will act to control the torque within a certain range. Similar to the simulation results, both $\alpha$ and $\beta$ have a very important influence on the actual control effect. When tunable parameter $\alpha$ and $\beta$ increase, the error convergence time will be shortened and the corresponding torque output will be more drastic, and the steady-state accuracy is affected to some extent. Therefore, the parameters adjustment should be balanced between convergence time and steady-state precision as well as the endurance capacity of the manipulator. 

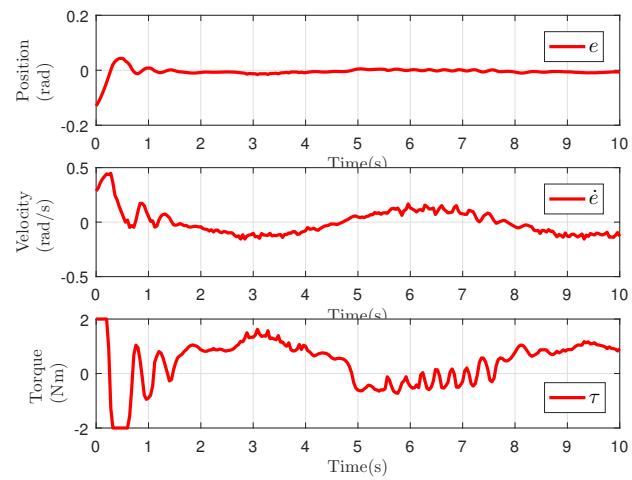

Fig. 17: Periodic tracking error and torque input by (23)
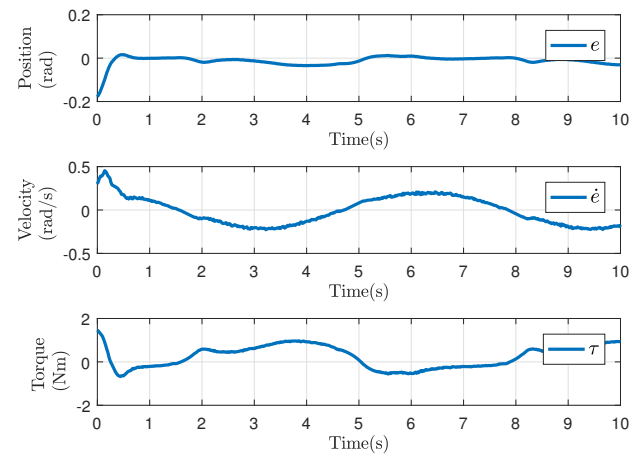

Fig. 18: Periodic tracking error and torque input by (38)

\section{CONCLUSIONS}

The problem of controlling the robot manipulator systems with unknown disturbances and actuator saturation has been discussed. A fixed-time convergent control algorithm combining non-singular terminal sliding mode control with RL is designed. The state of closed-loop system converges at fixed time under non-singular terminal sliding mode technique. Reinforcement learning control enables the robot to perform better learning ability with little environmental information. The critic NN is used to approximate the cost function of the system, and the actor NN is used in combination with the critic NN to design the appropriate control input for the manipulator. Considering the saturation of actuator, a nonlinear anti-windup compensator is designed to compensate for the saturation effect of the joint torque actuator. Simulations and experiments show that the system with the proposed control law can track the desired trajectory with good performance within a fixed time. In the future, the application of RL technology to other complex flexible structures will be a worthwhile research direction.

\section{REFERENCES}

[1] K. Hirai, M. Hirose, Y. Haikawa and T. Takenaka, "The development of honda humanoid robot." IEEE International Conference on Robotics and Automation, vol. 2, pp. 1321-1326, 1998.

[2] Z. Zuo, M. Defoort, B. Tian, Z. Ding, "Distributed consensus observer for multiagent systems with high-order integrator dynamics," IEEE Transactions on Automatic Control, vol. 65, no. 4, pp. 1771-1778, 2020.
[3] D. Zhao, S. Li, Q. Zhu and F. Gao, "Robust finite-time control approach for robotic manipulators," IET Control Theory \& Applications, vol. 4, no. 1, pp. 1-15, Jan. 2010.

[4] L. Zhao, B. Zhang, H. Yang and Y. Wang, "Finite-time tracking control for pneumatic servo system via extended state observer," IET Control Theory \& Applications, vol. 11, no. 16, pp. 2808-2816, 2017.

[5] L. Sun, and Y. Liu, "Extended state observer augmented finite-time trajectory tracking control of uncertain mechanical systems," Mechanical Systems and Signal Processing, vol. 11, no. 106374, 2019.

[6] L. Sun, and Z. Zheng, "Finite-time sliding mode trajectory tracking control of uncertain mechanical systems," Asian Journal of Control, vol. 19, no. 1, pp. 399-404, Jan. 2017.

[7] Y. Yang, et al, "Robust adaptive uniform exact tracking control for uncertain Euler-Lagrange system.” International Journal of Control, vol. 90, no. 12, pp. 2711-2720, 2017.

[8] S. Mondal, and C. Mahanta, "Adaptive second order terminal sliding mode controller for robotic manipulators," Journal of The Franklin Institute-Engineering and Applied Mathematics, vol. 351.4, pp. 23562377, 2014

[9] S. Bhat and D. S. Bernstein, "Finite-time stability of continuous autonomous systems," SIAM Journal on Control and Optimization, vol. 38, no. 3, pp. 751-766, 2000.

[10] M. V. Basin, P. C. R. Ramírez and F. Guerra-Avellaneda, "Continuous fixed-time controller design for mechatronic systems with incomplete measurements," IEEE/ASME Transactions on Mechatronics, vol. 23, no. 1, pp. 57-67, Feb. 2018.

[11] X. Jin, "Adaptive fixed-time control for MIMO nonlinear systems with asymmetric output constraints using universal barrier functions," IEEE Transactions on Automatic Control, vol. 64, no. 7, pp. 3046-3053, Jul. 2019.

[12] X. Liu and X. Liao, "Fixed-time H-infinity control for port-controlled Hamiltonian systems," IEEE Transactions on Automatic Control, vol. 64, no. 7, pp. 2753-2765, Jul. 2019.

[13] M. V. Basin, P. Yu and Y. B. Shtessel, "Hypersonic missile adaptive sliding mode control using finite- and fixed-time observers," IEEE Transactions on Industrial Electronics, vol. 65, no. 1, pp. 930-941, Jan. 2018.

[14] L. Sun and Y. Liu. "Fixed-time adaptive sliding mode trajectory tracking control of uncertain mechanical systems," Asian Journal of Control, pp. 2080-2089, 2019.

[15] Z. Zuo, B. Tian, M. Defoort and Z. Ding, "Fixed-time consensus tracking for multiagent systems with high-order integrator dynamics," IEEE Transactions on Automatic Control. vol. 63, no. 2, pp. 563-570, Feb. 2018.

[16] J. Li, Y. Yang, C. Hua and X. Guan, "Fixed-time backstepping control design for high-order strict-feedback non-linear systems via terminal sliding mode," IET Control Theory \& Applications, vol. 11, no. 8, pp. 1184-1193, 2017.

[17] B. Jiang, Q. Hu and M. I. Friswell, "Fixed-time attitude control for rigid spacecraft with actuator saturation and faults," IEEE Transactions on Control Systems Technology, vol. 24, no. 5, pp. 1892-1898, Sept. 2016.

[18] Z. Zhang and Y. Wu, "Fixed-time regulation control of uncertain nonholonomic systems and its applications," International Journal of Control, vol. 90, no. 7, pp. 1327-1344, July. 2017.

[19] M. V. Basin, F. Guerra-Avellaneda and Y. B. Shtessel, "Stock management problem: adaptive fixed-time convergent continuous controller design," IEEE Transactions on Systems, Man, and Cybernetics: Systems, vol. 50, no. 12, pp. 4974-4983, Dec. 2020.

[20] Z. Zuo, J. Song, B. Tian and M. Basin, "Robust fixed-time stabilization control of generic linear systems with mismatched disturbances," IEEE Transactions on Systems, Man, and Cybernetics: Systems, 2020.

[21] C. Yang, Z. Li, R. Cui and B. Xu, "Neural network-based motion control of an underactuated wheeled inverted pendulum model," IEEE Transactions on Neural Networks and Learning Systems, vol. 25, no. 11, pp. 2004-2016, Nov. 2014.

[22] P. Zhang, Z. Wu, H. Dong, M. Tan and J. Yu, "Reaction-wheel-based roll stabilization for a robotic fish using neural network sliding mode control," IEEE/ASME Transactions on Mechatronics, vol. 25, no. 4, pp. 1904-1911, Aug. 2020.

[23] W. He, S. S. Ge, Y. Li, E. Chew and Y. S. Ng, "Neural network control of a rehabilitation robot by state and output feedback," Journal of Intelligent \& Robotic Systems, vol. 80, pp. 15-31, Oct. 2015.

[24] C. Yang, Y. Jiang, Z. Li, W. He and C. Sun, "Neural control of bimanual robots with guaranteed global stability and motion precision," IEEE Transactions on Industrial Informatics, vol. 13, no. 3, pp. 1162-1171, June. 2017. 
[25] M. B Radac, A. I Borlea, "Virtual state feedback reference tuning and value iteration reinforcement learning for unknown observable systems control," Energies, vol. 14, no. 4, 1006, 2021.

[26] A. Perrusquía and W. Yu, "Discrete-Time Neural Control Using Reinforcement Learning," IEEE Transactions on Neural Networks and Learning Systems, doi: 10.1109/TNNLS.2020.3026010.

[27] W. He, H. Gao, C. Zhou, C. Yang and Z. Li, "Reinforcement learning control of a flexible two-link manipulator: an experimental investigation," IEEE Transactions on Systems, Man, and Cybernetics: Systems, doi: 10.1109/TSMC.2020.2975232.

[28] K G. Vamvoudakis and F L. Lewis, "Online actor-critic algorithm to solve the continuous-time infinite horizon optimal control problem," Automatica, vol. 46, no. 5, pp. 878-888, 2010

[29] H. An and B. Xian, "Attitude reinforcement learning control of an unmanned helicopter with verification," Control Theory \& Applications, vol. 35, No. 4, pp. 516-524, 2018.

[30] T, Li, Y. J. Liu and S. Tong, "Adaptive neural control using reinforcement learning for a class of robot manipulator," Neural Computing and Applications. vol. 25, no. 1, pp. 135-141, 2014.

[31] G. Wen, S. S. Ge and F. Tu, "Optimized backstepping for tracking control of strict-feedback systems," IEEE Transactions on Neural Networks and Learning Systems. vol. 29, no. 8, pp. 3850-3862, Aug. 2018.

[32] Y. Ouyang, W. He and X. Li, "Reinforcement learning control of a single-link flexible robotic manipulator," IET Control Theory and Applications. vol. 11, no. 9, pp. 1426-1433, 2017.

[33] X. Chen and Y. Yang "A novel adaptive PID controller based on ActorCritic learning," Control Theory \& Applications. vol. 28, no. 08, pp. 1187-1192, 2011.

[34] H. Liu, Y. Zhang and W. Wu, "Saturated adaptive back-stepping control for robot manipulators with RBF neural network approximation," IEEE International Conference on Information and Automation (ICIA). Ningbo, pp. 1550-1555, 2016.

[35] S. Ge, T. Lee and C. Harris, "Adaptive neural network control of robotic manipulators," World Scientific Publishing. December, 1998.

[36] Q. Guo, X. Li, Z. Zuo, Y. Shi and D. Jiang, "Quasi-synchronization control of multiple electro-hydraulic actuators with load disturbance and uncertain parameters," IEEE/ASME Transactions on Mechatronics. doi 10.1109/TMECH.2020.

[37] Z. Chen, F. Huang, W. Sun, J. Gu and B. Yao, "RBF-neural-networkbased adaptive robust control for nonlinear bilateral teleoperation manipulators with uncertainty and time delay," IEEE/ASME Transactions on Mechatronics, vol. 25, no. 2, pp. 906-918, April 2020.

[38] Z. Zuo and L. Tie, "A new class of finite-time nonlinear consensus protocols for multi-agent systems," International Journal of Control, vol. 87, no. 2, pp. 363-370, 2014.

[39] A. Polyakov, "Nonlinear feedback design for fixed-time stabilization of linear control systems," IEEE Transactions on Automatic Control. vol. 57, no. 8, pp. 2106-2110, Aug. 2012.

[40] Y Huang, Y Jia. Fixed-time consensus tracking control for second-order multi-agent systems with bounded input uncertainties via NFFTSM. IET Control Theory \& Applications, vol. 11, no. 16, pp. 2900-2909, 2017.

[41] M. Tran, H. Kang, "A novel adaptive finite-time tracking control for robotic manipulators using nonsingular terminal sliding mode and RBF neural networks," International Journal of Precision Engineering and Manufacturing, vol. 17, pp. 863-870, 2016.

\section{APPENDIX}

Select a candidate Lyapunov function as

$$
V(t)=\frac{1}{2} \boldsymbol{s}^{\mathrm{T}} \boldsymbol{s}+\frac{1}{2} \boldsymbol{\zeta}^{\mathrm{T}} \boldsymbol{\zeta}+\frac{1}{2 \delta_{c}} \tilde{\boldsymbol{w}}_{c}^{\mathrm{T}} \tilde{\boldsymbol{w}}_{c}+\frac{1}{2 \delta_{a}} \sum_{i=1}^{n} \tilde{\boldsymbol{w}}_{a i}^{\mathrm{T}} \tilde{\boldsymbol{w}}_{a i}
$$

Substituting (5), (8) and (23) into (21), the closed-loop system can be expressed as

$$
\begin{aligned}
\dot{\boldsymbol{s}}= & -\operatorname{sig}^{v_{4}}\left(\sigma_{1} \operatorname{sig}^{v_{2}}(\boldsymbol{s})+\sigma_{2} \operatorname{sig}^{v_{3}}(\boldsymbol{s})\right)-\boldsymbol{\zeta}-K_{s} \boldsymbol{s} \\
& +v_{1} E_{2}\left[M_{0}^{-1} \boldsymbol{\tau}_{\Delta}+\tilde{\boldsymbol{f}}+M_{0}^{-1} \boldsymbol{d}-\bar{d} \operatorname{sgn}(\boldsymbol{s})\right]
\end{aligned}
$$

where $\tilde{f}$ is the estimated error between the estimated value of RBFNN and optimal value, that is

$$
\tilde{\boldsymbol{f}}=\left[\begin{array}{c}
\boldsymbol{w}_{a 1}^{* \mathrm{~T}} \boldsymbol{\sigma}_{a 1}\left(\boldsymbol{z}_{a 1}\right) \\
\boldsymbol{w}_{a 2}^{* \mathrm{~T}} \boldsymbol{\sigma}_{a 2}\left(\boldsymbol{z}_{a 2}\right) \\
\ldots \\
\boldsymbol{w}_{a n}^{* \mathrm{~T}} \boldsymbol{\sigma}_{a n}\left(\boldsymbol{z}_{a n}\right)
\end{array}\right]-\left[\begin{array}{c}
\hat{\boldsymbol{w}}_{a 1}^{\mathrm{T}} \boldsymbol{\sigma}_{a 1}\left(\boldsymbol{z}_{a 1}\right) \\
\hat{\boldsymbol{w}}_{a 2}^{\mathrm{T}} \boldsymbol{\sigma}_{a 2}\left(\boldsymbol{z}_{a 2}\right) \\
\ldots \\
\hat{\boldsymbol{w}}_{a n}^{\mathrm{T}} \boldsymbol{\sigma}_{a n}\left(\boldsymbol{z}_{a n}\right)
\end{array}\right]+\left[\begin{array}{c}
\varepsilon_{1} \\
\varepsilon_{2} \\
\ldots \\
\varepsilon_{n}
\end{array}\right]
$$

and $\varepsilon_{i}(i=1,2, \cdots, n)$ are approximation error. Assume that $\varepsilon_{i} \leq k_{\varepsilon i}$ with small positive constants $k_{\varepsilon i}(i=1,2, \cdots, n)$.

Using (24), (40), the time derivative of $V(t)$ is

$$
\begin{aligned}
& \dot{V}(t)=\boldsymbol{s}^{\mathrm{T}} \dot{\boldsymbol{s}}+\boldsymbol{\zeta}^{\mathrm{T}} \dot{\boldsymbol{\zeta}}-\frac{1}{\delta_{c}} \tilde{\boldsymbol{w}}_{c}^{\mathrm{T}} \dot{\hat{\boldsymbol{w}}}_{c}-\frac{1}{\delta_{a}} \sum_{i=1}^{n} \tilde{\boldsymbol{w}}_{a i}^{\mathrm{T}} \dot{\boldsymbol{\boldsymbol { w }}}_{a i} \\
& \leq-\boldsymbol{s}^{\mathrm{T}} \operatorname{sig}^{v_{4}}\left(\sigma_{1} \operatorname{sig}^{v_{2}}(\boldsymbol{s})+\sigma_{2} \operatorname{sig}^{v_{3}}(\boldsymbol{s})\right)-\boldsymbol{s}^{\mathrm{T}} K_{s} \boldsymbol{s} \\
& -\boldsymbol{\zeta}^{\mathrm{T}} \operatorname{sig}^{v_{4}}\left(\sigma_{3} \operatorname{sig}^{v_{2}}(\boldsymbol{\zeta})+\sigma_{4} \operatorname{sig}^{v_{3}}(\boldsymbol{\zeta})\right)-\boldsymbol{\zeta}^{\mathrm{T}} K_{\zeta} \boldsymbol{\zeta} \\
& +v_{1} \boldsymbol{s}^{\mathrm{T}} E_{2} M_{0}^{-1} \boldsymbol{\tau}_{\Delta}+\boldsymbol{\zeta}^{\mathrm{T}} \boldsymbol{\tau}_{\Delta} \\
& +v_{1} \boldsymbol{s}^{\mathrm{T}} E_{2} \tilde{\boldsymbol{f}}-\frac{1}{\delta_{c}} \tilde{\boldsymbol{w}}_{c}^{\mathrm{T}} \dot{\boldsymbol{\boldsymbol { w }}}_{c}-\frac{1}{\delta_{a}} \sum_{i=1}^{n} \tilde{\boldsymbol{w}}_{a i}^{\mathrm{T}} \dot{\hat{\boldsymbol{w}}}_{a i}
\end{aligned}
$$

According to Young's inequality, one has $v_{1} \boldsymbol{s}^{\mathrm{T}} E_{2} M_{0}^{-1} \boldsymbol{\tau}_{\Delta} \leq \frac{v_{1}}{2} \boldsymbol{s}^{\mathrm{T}} K_{M} \boldsymbol{s}+\frac{v_{1}}{2} \boldsymbol{\tau}_{\Delta}^{\mathrm{T}} \boldsymbol{\tau}_{\Delta} \quad$ where $K_{M}=E_{2} M_{0}^{-1} M_{0}^{-1} E_{2}$. Similarly $\boldsymbol{\zeta}^{\mathrm{T}} \boldsymbol{\tau}_{\Delta} \leq \frac{1}{2} \boldsymbol{\zeta}^{\mathrm{T}} \boldsymbol{\zeta}+\frac{1}{2} \boldsymbol{\tau}_{\Delta}^{\mathrm{T}} \boldsymbol{\tau}_{\Delta}$. Using equation (41) and $\varepsilon_{i} \leq k_{\varepsilon i}$, we can obtain that $\varepsilon_{i}+\left\|\tilde{\boldsymbol{w}}_{a i}\right\| \leq k_{\varepsilon i}+\left\|w_{a i}\right\|+\left\|\bar{w}_{a i}\right\|=k_{\varepsilon w i}$. Substituting above inequality into (42), one has

$$
\begin{aligned}
\dot{V}(t) \leq & \left(\frac{1+v_{1}}{2}\right) \boldsymbol{\tau}_{\Delta}^{\mathrm{T}} \boldsymbol{\tau}_{\Delta}-\frac{1}{\delta_{c}} \tilde{\boldsymbol{w}}_{c}^{\mathrm{T}} \dot{\boldsymbol{\boldsymbol { w }}}_{c}-\frac{1}{\delta_{a}} \sum_{i=1}^{n} \tilde{\boldsymbol{w}}_{a i}^{\mathrm{T}} \dot{\boldsymbol{\boldsymbol { w }}}_{a i} \\
& -\boldsymbol{\zeta}^{\mathrm{T}}\left(K_{\zeta}-\frac{1}{2} E_{n}\right) \boldsymbol{\zeta}-\boldsymbol{s}^{\mathrm{T}} \operatorname{sig}^{v_{4}}\left(\sigma_{1} \operatorname{sig}^{v_{2}}(\boldsymbol{s})+\sigma_{2} \operatorname{sig}^{v_{3}}(\boldsymbol{s})\right) \\
& -\boldsymbol{\zeta}^{\mathrm{T}} \operatorname{sig}^{v_{4}}\left(\sigma_{3} \operatorname{sig}^{v_{2}}(\boldsymbol{\zeta})+\sigma_{4} \operatorname{sig}^{v_{3}}(\boldsymbol{\zeta})\right) \\
& -\left(\lambda_{m}\left(K_{s}\right)-\frac{1}{2} v_{1}\left\|K_{M}\right\|\right)\left(\|\boldsymbol{s}\|-\frac{v_{1}\left\|E_{2}\right\| \lambda_{M}\left(K_{\varepsilon w}\right)}{2 \lambda_{m}\left(K_{s}\right)-v_{1}\left\|K_{M}\right\|}\right)^{2} \\
& +\frac{\left[v_{1}\left\|E_{2}\right\| \lambda_{M}\left(K_{\varepsilon w}\right)\right]^{2}}{4\left(\lambda_{m}\left(K_{s}\right)-\frac{1}{2} v_{1}\left\|K_{M}\right\|\right)}
\end{aligned}
$$

where $K_{\varepsilon w}=\operatorname{diag}\left\{k_{\varepsilon w 1}, k_{\varepsilon w 2}, \cdots, k_{\varepsilon w n}\right\}$ is a diagonal positive definite matrix, $E_{n}$ is $n$ order identity matrix. Selecting the proper parameters ensure that $K_{\zeta}-\frac{1}{2} E_{n} \geq 0$ and $\lambda_{m}\left(K_{s}\right)-\frac{1}{2} v_{1}\left\|K_{M}\right\|>0$, by assigning $K_{s}=v_{1} K_{M}$, one has

$$
\begin{aligned}
\dot{V}(t) \leq & -\boldsymbol{s}^{\mathrm{T}} \operatorname{sig}^{v_{4}}\left(\sigma_{1} \operatorname{sig}^{v_{2}}(\boldsymbol{s})+\sigma_{2} \operatorname{sig}^{v_{3}}(\boldsymbol{s})\right) \\
& -\boldsymbol{\zeta}^{\mathrm{T}} \operatorname{sig}^{v_{4}}\left(\sigma_{3} \operatorname{sig}^{v_{2}}(\boldsymbol{\zeta})+\sigma_{4} \operatorname{sig}^{v_{3}}(\boldsymbol{\zeta})\right) \\
& +\left(\frac{1+v_{1}}{2}\right) \boldsymbol{\tau}_{\Delta}^{\mathrm{T}} \boldsymbol{\tau}_{\Delta}-\frac{1}{\delta_{c}} \tilde{\boldsymbol{w}}_{c}^{T} \dot{\hat{\boldsymbol{w}}}_{c} \\
& -\frac{1}{\delta_{a}} \sum_{i=1}^{n} \tilde{\boldsymbol{w}}_{a i}^{T} \dot{\hat{\boldsymbol{w}}}_{a i}+\frac{\left[v_{1}\left\|E_{2}\right\| \lambda_{M}\left(K_{\varepsilon w}\right)\right]^{2}}{4\left(\lambda_{m}\left(K_{s}\right)-\frac{1}{2} v_{1}\left\|K_{M}\right\|\right)}
\end{aligned}
$$

Remark 7: It can be seen from the above derivations that the choice of control gain $K_{s}$ can affect the steady-state accuracy of the system. For the sake of theoretical analysis, $K_{s}=v_{1} K_{M}$ is set as a time-varying matrix. In the actual simulation, $K_{s}$ can be set to a larger constant matrix, but overlarge control gain will excite high-frequency dynamics of robot system. The control gain matrix $K_{s}$ can be obtained by using trial-and-error method. 
When $\left\|\hat{\boldsymbol{w}}_{c}\right\| \leq\left\|\overline{\boldsymbol{w}}_{c}\right\|$ or $\left\|\hat{\boldsymbol{w}}_{c}\right\|=\left\|\overline{\boldsymbol{w}}_{c}\right\|, \hat{\boldsymbol{w}}_{c}^{\mathrm{T}} \boldsymbol{\rho}_{c}>0$; $\left\|\hat{\boldsymbol{w}}_{a i}\right\| \leq\left\|\overline{\boldsymbol{w}}_{a i}\right\|$ or $\left\|\hat{\boldsymbol{w}}_{a i}\right\|=\left\|\overline{\boldsymbol{w}}_{a i}\right\|, \hat{\boldsymbol{w}}_{a i}^{\mathrm{T}} \boldsymbol{\rho}_{a i}>0$. Substituting (18) and (31) into (44), one has

$$
\begin{aligned}
\dot{V}(t) \leq & -\boldsymbol{s}^{\mathrm{T}} \operatorname{sig}^{v_{4}}\left(\sigma_{1} \operatorname{sig}^{v_{2}}(\boldsymbol{s})+\sigma_{2} \operatorname{sig}^{v_{3}}(\boldsymbol{s})\right) \\
& -\boldsymbol{\zeta}^{\mathrm{T}} \operatorname{sig}^{v_{4}}\left(\sigma_{3} \operatorname{sig}^{v_{2}}(\boldsymbol{\zeta})+\sigma_{4} \operatorname{sig}^{v_{3}}(\boldsymbol{\zeta})\right) \\
& +\sum_{i=1}^{n} \tilde{\boldsymbol{w}}_{a i}^{\mathrm{T}} \boldsymbol{\rho}_{a i}+\tilde{\boldsymbol{w}}_{c}^{\mathrm{T}} \boldsymbol{\rho}_{c}+\left(\frac{1+v_{1}}{2}\right) \boldsymbol{\tau}_{\Delta}^{\mathrm{T}} \boldsymbol{\tau}_{\Delta} \\
& +\frac{\left[v_{1}|| E_{2}|| \lambda_{M}\left(K_{\varepsilon w}\right)\right]^{2}}{4\left(\lambda_{m}\left(K_{s}\right)-\frac{1}{2} v_{1}\left\|K_{M}\right\|\right)} \\
= & -\sum_{i=1}^{n}\left(\left(\sigma_{1}\left(\left|s_{i}\right|^{2}\right)^{\left(1+v_{2} v_{4}\right) / 2 v_{4}}+\sigma_{2}\left(\left|s_{i}\right|^{2}\right)^{\left(1+v_{3} v_{4}\right) / 2 v_{4}}\right)^{v_{4}}\right. \\
& \left.+\left(\sigma_{3}\left(\left|\zeta_{i}\right|^{2}\right)^{\left(1+v_{2} v_{4}\right) / 2 v_{4}}+\sigma_{4}\left(\left|\zeta_{i}\right|^{2}\right)^{\left(1+v_{3} v_{4}\right) / 2 v_{4}}\right)^{v_{4}}\right)+\Omega_{1} \\
\leq & -\sum_{i=1}^{n}\left(\left(\sigma_{1}\left(\left|s_{i}\right|^{2}\right)^{\left(1+v_{2} v_{4}\right) / 2 v_{4}}+\sigma_{2}\left(\left|s_{i}\right|^{2}\right)^{\left(1+v_{3} v_{4}\right) / 2 v_{4}}\right)^{v_{4}}\right. \\
& \left.+\left(\sigma_{3}\left(\left|\zeta_{i}\right|^{2}\right)^{\left(1+v_{2} v_{4}\right) / 2 v_{4}}+\sigma_{4}\left(\left|\zeta_{i}\right|^{2}\right)^{\left(1+v_{3} v_{4}\right) / 2 v_{4}}\right)^{v_{4}}\right)+\bar{\Omega}_{1}
\end{aligned}
$$

where $\Omega_{1}=\sum_{i=1}^{n} \tilde{\boldsymbol{w}}_{a i}^{\mathrm{T}} \boldsymbol{\rho}_{a i}+\tilde{\boldsymbol{w}}_{c}^{\mathrm{T}} \boldsymbol{\rho}_{c}+\frac{\left[v_{1}\left\|E_{2}\right\| \lambda_{M}\left(K_{\varepsilon w}\right)\right]^{2}}{4\left(\lambda_{m}\left(K_{s}\right)-\frac{1}{2} v_{1}\left\|K_{M}\right\|\right)}+$ $\left(\frac{1+v_{1}}{2}\right) \tau_{\Delta}^{\mathrm{T}} \tau_{\Delta}, \bar{\Omega}_{1}=n\left(\left\|w_{a i}^{*}\right\|+\left\|\bar{w}_{a i}\right\|\right)+\frac{\left[v_{1}\left\|E_{2}\right\| \lambda_{M}\left(K_{\varepsilon w}\right)\right]^{2}}{4\left(\lambda_{m}\left(K_{s}\right)-\frac{1}{2} v_{1}\left\|K_{M}\right\|\right)}+$ $\left(1+\left\|\bar{w}_{c}\right\|\|\Lambda\|\right)\left(\left\|w_{c}^{*}\right\|+\left\|\bar{w}_{c}\right\|\right)\|\Lambda\|+\left(\frac{1+v_{1}}{2}\right) \bar{\tau}_{\Delta}^{2}$.

When $\left\|\hat{\boldsymbol{w}}_{c}\right\|=\left\|\overline{\boldsymbol{w}}_{c}\right\|, \boldsymbol{w}_{c}^{\mathrm{T}} \boldsymbol{\rho}_{c} \leq 0 ;\left\|\hat{\boldsymbol{w}}_{a i}\right\|=$ $\left\|\overline{\boldsymbol{w}}_{a i}\right\|, \boldsymbol{w}_{a i}^{\mathrm{T}} \boldsymbol{\rho}_{a i} \leq 0$. Substituting (18) and (31) into (44), one has

$$
\begin{aligned}
\dot{V}(t) \leq & -\boldsymbol{s}^{\mathrm{T}} \operatorname{sig}^{v_{4}}\left(\sigma_{1} \operatorname{sig}^{v_{2}}(\boldsymbol{s})+\sigma_{2} \operatorname{sig}^{v_{3}}(\boldsymbol{s})\right) \\
& -\boldsymbol{\zeta}^{\mathrm{T}} \operatorname{sig}^{v_{4}}\left(\sigma_{3} \operatorname{sig}^{v_{2}}(\boldsymbol{\zeta})+\sigma_{4} \operatorname{sig}^{v_{3}}(\boldsymbol{\zeta})\right) \\
& +\frac{\left[v_{1} \| E_{2}|| \lambda_{M}\left(K_{\varepsilon w}\right)\right]^{2}}{4\left(\lambda_{m}\left(K_{s}\right)-\frac{1}{2} v_{1}\left\|K_{M}\right\|\right)}+\left(\frac{1+v_{1}}{2}\right) \boldsymbol{\tau}_{\Delta}^{\mathrm{T}} \boldsymbol{\tau}_{\Delta} \\
= & -\sum_{i=1}^{n}\left(\left(\sigma_{1}\left(\left|s_{i}\right|^{2}\right)^{\left(1+v_{2} v_{4}\right) / 2 v_{4}}+\sigma_{2}\left(\left|s_{i}\right|^{2}\right)^{\left(1+v_{3} v_{4}\right) / 2 v_{4}}\right)^{v_{4}}\right. \\
& \left.+\left(\sigma_{3}\left(\left|\zeta_{i}\right|^{2}\right)^{\left(1+v_{2} v_{4}\right) / 2 v_{4}}+\sigma_{4}\left(\left|\zeta_{i}\right|^{2}\right)^{\left(1+v_{3} v_{4}\right) / 2 v_{4}}\right)^{v_{4}}\right)+\Omega_{1} \\
\leq & -\sum_{i=1}^{n}\left(\left(\sigma_{1}\left(\left|s_{i}\right|^{2}\right)^{\left(1+v_{2} v_{4}\right) / 2 v_{4}}+\sigma_{2}\left(\left|s_{i}\right|^{2}\right)^{\left(1+v_{3} v_{4}\right) / 2 v_{4}}\right)^{v_{4}}\right. \\
& \left.+\left(\sigma_{3}\left(\left|\zeta_{i}\right|^{2}\right)^{\left(1+v_{2} v_{4}\right) / 2 v_{4}}+\sigma_{4}\left(\left|\zeta_{i}\right|^{2}\right)^{\left(1+v_{3} v_{4}\right) / 2 v_{4}}\right)^{v_{4}}\right)+\bar{\Omega}_{1}
\end{aligned}
$$

where $\Omega_{1}=\frac{\left[v_{1}\left\|E_{2}\right\| \lambda_{M}\left(K_{\varepsilon w}\right)\right]^{2}}{4\left(\lambda_{m}\left(K_{s}\right)-\frac{1}{2} v_{1}\left\|K_{M}\right\|\right)}+\left(\frac{1+v_{1}}{2}\right) \tau_{\Delta}^{\mathrm{T}} \boldsymbol{\tau}_{\Delta}, \bar{\Omega}_{1}=$ $\frac{\left[v_{1}\left\|E_{2}\right\| \lambda_{M}\left(K_{\varepsilon w}\right)\right]^{2}}{4\left(\lambda_{m}\left(K_{s}\right)-\frac{1}{2} v_{1}\left\|K_{M}\right\|\right)}+\left(\frac{1+v_{1}}{2}\right) \bar{\tau}_{\Delta}^{2}$.

Remark 8: The above equations (45) and (46) discuss the two cases respectively. There are two other cases $\left\|\hat{\boldsymbol{w}}_{c}\right\| \leq\left\|\overline{\boldsymbol{w}}_{c}\right\|$ or $\left\|\hat{\boldsymbol{w}}_{c}\right\|=\left\|\overline{\boldsymbol{w}}_{c}\right\|, \hat{\boldsymbol{w}}_{c}^{\mathrm{T}} \boldsymbol{\rho}_{c}>0 ;\left\|\hat{\boldsymbol{w}}_{a i}\right\|=$ $\left\|\overline{\boldsymbol{w}}_{a i}\right\|, \boldsymbol{w}_{a i}^{\mathrm{T}} \boldsymbol{\rho}_{a i} \leq 0$ and $\left\|\hat{\boldsymbol{w}}_{c}\right\|=\left\|\overline{\boldsymbol{w}}_{c}\right\|, \boldsymbol{w}_{c}^{\mathrm{T}} \boldsymbol{\rho}_{c} \leq 0$; $\left\|\hat{\boldsymbol{w}}_{a i}\right\| \leq\left\|\overline{\boldsymbol{w}}_{a i}\right\|$ or $\left\|\hat{\boldsymbol{w}}_{a i}\right\|=\left\|\overline{\boldsymbol{w}}_{a i}\right\|, \hat{\boldsymbol{w}}_{a i}^{\mathrm{T}} \boldsymbol{\rho}_{a i}>0$ and can easily get the same result, we will not do too much proof here.

Then, by adding some auxiliary terms, equation (45) can be simplified as

$$
\begin{aligned}
\dot{V}(t) \leq & -\sum_{i=1}^{n}\left(\left(\sigma_{1}\left(\left|s_{i}\right|^{2}\right)^{\left(1+v_{2} v_{4}\right) / 2 v_{4}}+\sigma_{2}\left(\left|s_{i}\right|^{2}\right)^{\left(1+v_{3} v_{4}\right) / 2 v_{4}}\right)^{v_{4}}\right. \\
& \left.+\left(\sigma_{3}\left(\left|\zeta_{i}\right|^{2}\right)^{\left(1+v_{2} v_{4}\right) / 2 v_{4}}+\sigma_{4}\left(\left|\zeta_{i}\right|^{2}\right)^{\left(1+v_{3} v_{4}\right) / 2 v_{4}}\right)^{v_{4}}\right)+\bar{\Omega}_{1} \\
& -\left(\frac{1}{\delta_{c}} \tilde{\boldsymbol{w}}_{c}^{\mathrm{T}} \tilde{\boldsymbol{w}}_{c}\right)^{\left(1+v_{2} v_{4}\right) / 2}+\left(\frac{1}{\delta_{c}} \tilde{\boldsymbol{w}}_{c}^{\mathrm{T}} \tilde{\boldsymbol{w}}_{c}\right)^{\left(1+v_{2} v_{4}\right) / 2} \\
& -\left(\frac{1}{\delta_{c}} \tilde{\boldsymbol{w}}_{c}^{\mathrm{T}} \tilde{\boldsymbol{w}}_{c}\right)^{\left(1+v_{3} v_{4}\right) / 2}+\left(\frac{1}{\delta_{c}} \tilde{\boldsymbol{w}}_{c}^{\mathrm{T}} \tilde{\boldsymbol{w}}_{c}\right)^{\left(1+v_{3} v_{4}\right) / 2} \\
& -\left(\frac{1}{\delta_{a}} \sum_{i=1}^{n} \tilde{\boldsymbol{w}}_{a i}^{\mathrm{T}} \tilde{\boldsymbol{w}}_{a i}\right)^{\left(1+v_{2} v_{4}\right) / 2} \\
& +\left(\frac{1}{\delta_{a}} \sum_{i=1}^{n} \tilde{\boldsymbol{w}}_{a i}^{\mathrm{T}} \tilde{\boldsymbol{w}}_{a i}\right)^{\left(1+v_{2} v_{4}\right) / 2} \\
& -\left(\frac{1}{\delta_{a}} \sum_{i=1}^{n} \tilde{\boldsymbol{w}}_{a i}^{\mathrm{T}} \tilde{\boldsymbol{w}}_{a i}\right)^{\left(1+v_{3} v_{4}\right) / 2} \\
& +\left(\frac{1}{\delta_{a}} \sum_{i=1}^{n} \tilde{\boldsymbol{w}}_{a i}^{\mathrm{T}} \tilde{\boldsymbol{w}}_{a i}\right)^{\left(1+v_{3} v_{4}\right) / 2}
\end{aligned}
$$

According to Lemma 1, equation (47) can be simplified as

$$
\begin{aligned}
\dot{V}(t) \leq & -(3 n+1)^{1-v_{4}}\left(\operatorname { m i n } \{ \sigma _ { 1 } , \sigma _ { 3 } , 1 \} \left(\sum_{i=1}^{n}\left|s_{i}\right|^{2}+\sum_{i=1}^{n}\left|\zeta_{i}\right|^{2}\right.\right. \\
& \left.+\frac{1}{\delta_{c}} \tilde{\boldsymbol{w}}_{c}^{\mathrm{T}} \tilde{\boldsymbol{w}}_{c}+\frac{1}{\delta_{a}} \sum_{i=1}^{n} \tilde{\boldsymbol{w}}_{a i}^{\mathrm{T}} \tilde{\boldsymbol{w}}_{a i}\right)^{\left(1+v_{2} v_{4}\right) / 2 v_{4}} \\
& +\min \left\{\sigma_{2}, \sigma_{4}, 1\right\}\left(\sum_{i=1}^{n}\left|s_{i}\right|^{2}+\sum_{i=1}^{n}\left|\zeta_{i}\right|^{2}\right. \\
& \left.\left.+\frac{1}{\delta_{c}} \tilde{\boldsymbol{w}}_{c}^{\mathrm{T}} \tilde{\boldsymbol{w}}_{c}+\frac{1}{\delta_{a}} \sum_{i=1}^{n} \tilde{\boldsymbol{w}}_{a i}^{\mathrm{T}} \tilde{\boldsymbol{w}}_{a i}\right)^{\left(1+v_{3} v_{4}\right) / 2 v_{4}}\right)^{v_{4}} \\
& +\Omega_{2} \\
= & -(3 n+1)^{1-v_{4}}\left(\sigma_{5} V^{\left(1+v_{2} v_{4}\right) / 2 v_{4}}+\sigma_{6} V^{\left(1+v_{3} v_{4}\right) / 2 v_{4}}\right)^{v_{4}}+\Omega_{2} \\
\leq & -(3 n+1)^{1-v_{4}}\left(\sigma_{5} V^{\left(1+v_{2} v_{4}\right) / 2 v_{4}}+\sigma_{6} V^{\left(1+v_{3} v_{4}\right) / 2 v_{4}}\right)^{v_{4}}+\bar{\Omega}_{2}
\end{aligned}
$$

where $\Omega_{2}=\left(\frac{1}{\delta_{c}} \tilde{\boldsymbol{w}}_{c}^{\mathrm{T}} \tilde{\boldsymbol{w}}_{c}\right)^{\left(1+v_{2} v_{4}\right) / 2}+\left(\frac{1}{\delta_{c}} \tilde{\boldsymbol{w}}_{c}^{\mathrm{T}} \tilde{\boldsymbol{w}}_{c}\right)^{\left(1+v_{3} v_{4}\right) / 2}+$ $\left(\frac{1}{\delta_{a}} \sum_{i=1}^{n} \tilde{\boldsymbol{w}}_{a i}^{\mathrm{T}} \tilde{\boldsymbol{w}}_{a i}\right)^{\left(1+v_{2} v_{4}\right) / 2}+\left(\frac{1}{\delta_{a}} \sum_{i=1}^{n} \tilde{\boldsymbol{w}}_{a i}^{\mathrm{T}} \tilde{\boldsymbol{w}}_{a i}\right)^{\left(1+v_{3} v_{4}\right) / 2}+\bar{\Omega}_{1}$, $\bar{\Omega}_{2}=\bar{\Omega}_{1}+\left(\delta_{c}^{-\frac{1}{2}}\left(\left\|w_{c}^{*}\right\|+\left\|\bar{w}_{c}\right\|\right)\right)^{1+v_{2} v_{4}}+$ $\left(\delta_{c}^{-\frac{1}{2}}\left(\left\|w_{c}^{*}\right\|+\left\|\bar{w}_{c}\right\|\right)\right)^{1+v_{3} v_{4}}+\left(n \delta_{a}^{-\frac{1}{2}}\left(\left\|w_{a i}^{*}\right\|+\right.\right.$ $\left.\left.\left\|\bar{w}_{a i}\right\|\right)\right)^{1+v_{2} v_{4}}+\left(n \delta_{c}^{-\frac{1}{2}}\left(\left\|w_{c i}^{*}\right\|+\left\|\bar{w}_{a i}\right\|\right)\right)^{1+v_{3} v_{4}}$, $\sigma_{5}=2^{\left(1+v_{2} v_{4}\right) / 2 v_{4}} \min \left\{\sigma_{1}, \sigma_{3}, 1\right\}, \sigma_{6}=$ $2^{\left(1+v_{3} v_{4}\right) / 2 v_{4}} \min \left\{\sigma_{2}, \sigma_{4}, 1\right\}$.

According to Lemma 2, by selecting appropriate parameters, the tracking errors $e_{1}$ and $e_{2}$ will be driven by the sliding mode to $s_{i}=\bar{s}(i=1,2, \cdots, n)$ in fixed time

$$
\begin{aligned}
T_{1} & =\frac{2}{(3 n+1)^{1-v_{4}} \sigma_{5}^{v_{4}} \theta^{v_{4}}\left(1-v_{2} v_{4}\right)} \\
& +\frac{2}{(3 n+1)^{1-v_{4}} \sigma_{6}^{v_{4}} \theta^{v_{4}}\left(v_{3} v_{4}-1\right)}
\end{aligned}
$$

where $\bar{s}=\left\{s_{i} \mid\|s\| \leq \min \left\{\frac{1}{\sigma_{5}^{v_{4}}}\left(\frac{\bar{\Omega}_{2}}{1-\theta}\right)^{\frac{2}{1+v_{2} v_{4}}}, \frac{1}{\sigma_{6}^{v_{4}}}\left(\frac{\bar{\Omega}_{2}}{1-\theta}\right)^{\frac{2}{1+v_{3} v_{4}}}\right\}\right\}$.

If $\bar{s}=k_{e_{1 i}} e_{1 i}+\operatorname{sig}^{v_{1}}\left(e_{2 i}\right)(i=1,2, \cdots, n)$, then

$$
\dot{e}_{1 i}=-\operatorname{sig}\left(\alpha \operatorname{sig}^{p}\left(e_{1 i}\right)+\beta \operatorname{sig}^{g}\left(e_{1 i}\right)\right)^{k}+\bar{s}^{\frac{1}{v_{1}}}
$$


Let the positive definite Lyapunov function be of the form $V_{\boldsymbol{e}_{1}}(t)=\sum_{i=1}^{n}\left|e_{1 i}\right|$. Taking the derivative of $V_{\boldsymbol{e}_{1}}$ along (49) yields

$$
\begin{aligned}
\dot{V}_{\boldsymbol{e}_{1}}(t) & =\sum_{i=1}^{n} \operatorname{sign}\left(e_{1 i}\right) \dot{e}_{1 i} \\
& =-\sum_{i=1}^{n}\left|\alpha \operatorname{sig}^{p}\left(e_{1 i}\right)+\beta \operatorname{sig}^{g}\left(e_{1 i}\right)\right|^{k}+\sum_{i=1}^{n} \operatorname{sign}\left(e_{1 i}\right) \bar{s}^{\frac{1}{v_{1}}} \\
& \leq-\left(\alpha V_{\boldsymbol{e}_{1}}{ }^{p}+\beta V_{\boldsymbol{e}_{1}}{ }^{g}\right)^{k}+\left|\bar{s}^{\frac{1}{v_{1}}}\right|
\end{aligned}
$$

According to Lemma 2, the tracking error $\boldsymbol{e}_{1}$ will converge to a certain vicinity of the origin $\bar{e}_{1}$ in fixed time, where $\bar{e}_{1}=\left\{\mathrm{x} \mid\|x\| \leq \min \left\{\frac{1}{\alpha^{k}}\left(\frac{\frac{1}{v_{1}}}{1-\theta}\right)^{\frac{1}{k p}}, \frac{1}{\beta^{k}}\left(\frac{\bar{s}^{\frac{1}{v_{1}}}}{1-\theta}\right)^{\frac{1}{k q}}\right\}\right\}$, because $e_{2 i}=-\operatorname{sig}\left(\alpha \operatorname{sig}^{p}\left(e_{1 i}\right)+\beta \operatorname{sig}^{g}\left(e_{1 i}\right)\right)^{k}+\bar{s}^{\frac{1}{v_{1}}}$, therefore $e_{2}$ will also converge to a certain vicinity of the origin.

Therefore, if the sliding surface $s$ converge to small neighborhood around zero, then the tracking errors $e_{1}$ and $e_{2}$ will converge to small neighborhoods around zero in fixed time, which is independent of the initial value of system states, and the upper bound of convergence time can be estimated as

$$
T_{2}=\frac{1}{\alpha^{k} \theta^{k}(1-p k)}+\frac{1}{\beta^{k} \theta^{k}(g k-1)}
$$

Further, it can be concluded that the tracking errors $e_{1}$ and $e_{2}$ will converge to small neighborhoods around zero in fixed time $T=T_{1}+T_{2}$. The proof is completed.

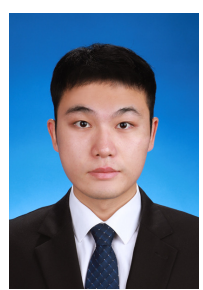

Shengjie CAO was born in 1997. He received the B.E. degree in Automatic Control from Henan Polytechnic University, Henan, China, in 2019. He is currently pursuing the M.E. degree with the School of Automation and Electrical Engineering, University of Science and Technology Beijing, Beijing, China. His current research interests include reinforcement learning, finite-time control, and robotics.

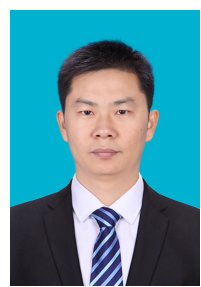

Liang Sun (M'15) received the M.Sc. and Ph.D. degrees in control theory and control engineering from Beihang University, China, in 2011 and 2016, respectively. He was a Postdoctoral Fellow in mechanical engineering with Beihang University from 2015 to 2017, and a Visiting Scholar with Loughborough University, UK, from 2019 to 2020 . He joined University of Science and Technology Beijing as an Associate Professor in 2017. His current research interests include nonlinear mechanical systems control, aerospace control and intelligent control.

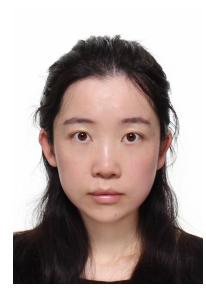

Jingjing Jiang (M'16) received the B.E. Degree in Electrical and Electronic Engineering from the University of Birmingham, UK, and the Harbin Institute of Technology, China, in 2010, the M.Sc. degree in Control Systems from Imperial College London, UK, in 2011, and the Ph.D degree from Imperial College London, in 2016. She carried out research as part of the Control and Power Group at Imperial College and joined Loughborough University as a Lecturer in September 2018. Her current research interests include driver assistance control and autonomous vehicle control design, control design of systems with constraints and human-in-the-loop.

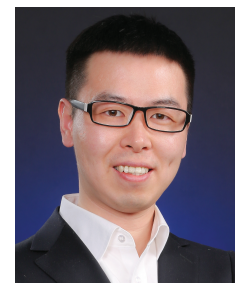

Zongyu Zuo (M'14-SM'18) received his B.Eng. degree in Automatic Control from Central South University, Hunan, China, in 2005, and Ph.D. degree in Control Theory and Applications from Beihang University (BUAA), Beijing, China, in 2011.

He was an academic visitor at the School of Electrical and Electronic Engineering, University of Manchester from September 2014 to September 2015 and held an inviting associate professorship at Mechanical Engineering and Computer Science, UMR CNRS 8201, Université de Valenciennes et du Hainaut-Cambrésis in October 2015 and May 2017. He is currently a full professor at the School of Automation Science and Electrical Engineering, Beihang University. His research interests are in the fields of nonlinear system control, control of UAVs, and coordination of multi-agent system. He was identified as a Highly Cited Researcher - 2020 by Clarivate Analytics.

Prof. Zuo serves as an Associate Editor of the Journal of The Franklin Institute, the Journal of Vibration and Control, the International Journal of Aeronautical \& Space Sciences, and the International Journal of Digital Signals and Smart Systems. 